\title{
Marc Lewon
}

\section{»Alexandre Agricola et ung bon joueur de luz« Agricola und die Laute}

In einem Brief aus dem Jahre 1492 schreibt König Karl VIII. von Frankreich an Piero de' Medici, den Sohn des gerade verstorbenen Lorenzo de' Medici:

Cher et amé cousin: Nous avons puis nagueres esté advertiz que Alexandre Agricola, chantre de nostre chappelle, et ung bon joueur de luz en sa compaignie arriverent ou temps de feu nostre cousin le Seigneur Laurens de Medicis, vostre pere, en la cité de Florence. Et pource que nous desirons singulierement recouvrer en nostre dicte chappelle icellui Alexandre, nous lui escripvons presentement qu'il s'en vienne devers nous et qu'il amene avec luy ledit joueur de luz ...

Donné a Poissy le XXV me jour d'avril.

Werter und geliebter Cousin: Wir wurden unlängst davon in Kenntnis gesetzt, dass Alexander Agricola, Sänger Unserer Kapelle, und ein guter Lautenist in seiner Gesellschaft während der (Regierungs-)Zeit Unseres kürzlich verstorbenen Cousins, des Herrn Lorenzo de' Medici, Eures Vaters, in der Stadt Florenz angekommen ist. Da Wir ausdrücklich die Rückkehr dieses Alexander an Unsere Kapelle wünschen, schreiben Wir ihm umgehend, damit er vor Uns trete und besagten Lautenisten mitbringe ...

Gegeben zu Poissy, am 25. Tag des April. ${ }^{1}$

Wenn man von diesem Zitat einmal absieht, so ist es zunächst schwer, Agricolas Euvre mit der Laute in eine direkte Verbindung zu bringen: Es sind keine Lautenkompositionen von ihm überliefert und es gibt keine Nachweise dafür, dass er dieses Instrument selbst gespielt, eigens dafür geschrieben oder es in Aufführungen seiner Musik eingesetzt hätte.

Zwar wird die Formulierung »clarus vocum manuumque« aus seinem Epitaph (»der Stimme und Hände wegen berühmt«) in der Regel dahingehend ausgelegt, dass Agricola berühmt für »seinen Gesang und sein Instrumentalspiel« war, er neben seiner Tätigkeit als Sänger also auch Instrumente beherrschte. ${ }^{2}$ Obwohl diese Deutung durchaus plausibel erscheint,

1 Übersetzt nach dem Zitat bei Martin Picker, »A letter of Charles VIII of France concerning Alexander Agricola«, in: Aspects of Medieval and Renaissance Music: A Birthday Offering to Gustave Reese, hrsg. von Jan LaRue, New York 1966, S. 668f.

2 Es wird bisweilen sogar soweit gegangen, aufgrund dieser Erwähnung anzunehmen, Agricola habe speziell Saiteninstrumente oder ganz konkret die Laute gespielt: »Given Agricola's apparent fame as a string player (he had been clarus vocum manuumque, according to the Epitaphion, and wrote several instrumental pieces), it is also possible that 
sollte man aber zumindest in Erwägung ziehen, dass die Formulierung sich auf die Disziplinen des »Musicus« und »Cantor« beziehen könnte, und gewählt wurde, um herauszustellen, dass Agricola die Fertigkeiten des praktizierenden Musikers ebenso wie die des gelehrten Musiktheoretikers und Komponisten $^{3}$ in seiner Person vereinigte, ohne damit auf spezifische instrumentale Fertigkeiten anzuspielen.

Auch aus obigem Brief ist nicht eindeutig ersichtlich, ob Agricola und der erwähnte Lautenist irgendetwas miteinander zu tun hatten - Agricola sich etwa begleiten ließ oder eine Aufführung mit dem Lautenisten gestaltete.

Andererseits war die Laute bereits während des gesamten 15. Jahrhunderts eines der beliebtesten und verbreitetsten Instrumente, wie eine reiche ikonographische Überlieferung und zahlreiche Nennungen bezeugen; und es war üblich, zeitgenössische Kompositionen darauf umzusetzen, wie man anhand der im späten 15. Jahrhundert einsetzenden Überlieferung von Lautentabulaturen sehen kann.

Auf der Suche nach einem Ansatz für die Verwendung der Laute im Werk Agricolas gerät man schnell an die gehäufte Überlieferung textloser Stücke, die im späten 15. Jahrhundert mit der Generation von Komponisten einsetzt, zu denen auch Agricola zählt, und die immer wieder Anlass zur Diskussion im Hinblick auf ihre Funktion und Besetzung geben: ob als genuine Instrumentalmusik konzipiert, eventuell sogar für ein bestimmtes Ensemble vorgesehen, $o b$ als rein kompositorische Idee veranlagt, ohne eine bestimmte, intendierte Besetzung, für die viele Aufführungsvarianten gleichwertig nebeneinander stehen - oder ob sie überhaupt für Instrumente gedacht waren.

he had been associated, from an early age, with the Ghent guild of soft-instrument players.« (Rob C. Wegman, »Agricola, Alexander«, in: The New Grove Dictionary of Music and Musicians, 2. Aufl., Bd. 1, London 2001, S. 225). Jon Banks, der sich ebenfalls auf die eingangs zitierte Passage aus dem Brief Karls VIII. bezieht, rückt die Deutung des Epitaphs in die Nähe des Lautenspiels, indem er mit Bezug auf die Textstelle schreibt: »... (>skilled in voice and hand $<$ ), and though his manual skills may have referred to the organ he was not above keeping company with lutenists.« (Jon Banks, The Instrumental Consort Repertory of the Late Fifteenth Century, Aldershot 2006, S. 40).

3 Dietrich Kämper erwähnt zwar eine Deutung Van der Straetens, der »die Möglichkeit in Betracht zieht, » >clarus ... manuum< ziele auf das Niederschreiben der Werke, also auf die kompositorische Tätigkeit ...«, schließt sich dann aber doch Van der Straetens persönlicher Meinung an, die Aussage weise auf Agricola als Lautenisten hin. (Dietrich Kämper, »Instrumentale Stilelemente bei Alexander Agricola«, in: Tijdschrift van de Vereniging voor Nederlandse Muziekgeschiedenis 28, 1978, S. 2; dort bezieht er sich auf Edmond van der Straeten, La musique aux Pays-Bas avant le XIX $X^{e}$ siècle, Bd. 7, Brüssel 1885, Reprint New York 1969, S. 133). 
Mittlerweile wird die Festlegung auf eine bestimmte Instrumentierung oder ein festes Ensemble weitgehend umgangen, als praktizierender Musiker muss man sich jedoch mit Besetzungsfragen auseinander setzen und sieht sich immer wieder vor dem Problem bezüglich der Funktion dieser Kompositionen.

An dieser Stelle setzt eine neue Monografie von Jon Banks an. In seinem Buch The Instrumental Consort Repertory of the Late Fifteenth Century ${ }^{4}$ legt Banks eine detaillierte Argumentationskette dar, in deren Verlauf er bestimmte textlos tradierte Stücke als Instrumentalrepertoire identifiziert, zusammenfasst, nach Genres sortiert, einen Aufführenden- und Rezipientenkreis dafür postuliert sowie eine angenommene instrumentale Besetzung rekonstruiert, für die das Repertoire verfasst worden sein könnte.

In dieses textlose Repertoire finden sowohl Stücke Eingang, die als instrumentale Kompositionen weitgehend anerkannt sind, weil sie weder Textincipits aufweisen, noch mit einer vokalen Gattung identifiziert werden können, sondern auch textlos überlieferte Chansons und Chansonbearbeitungen, also Kompositionen, die in direktem Zusammenhang mit zugrunde liegenden Texten sowie vokaler Konzeption und Aufführung stehen. Banks gliedert dieses Repertoire grob in drei Gruppen:

1. Textlose Chansons, die er auch in Übernahme des Begriffs von Warwick Edwards als $»$ Lieder ohne Worte $\varkappa^{5}$ bezeichnet. Diese Chansons sind in der Regel parallel zu ihrer textlosen Überlieferung in anderen Quellen auch textiert zu finden und entstammen eindeutig einer vokalen Gattung. Banks hält deren untextierte Niederschrift nicht für eine Art >unvollständiger< Überlieferung, sondern für einen sehr bewussten Schritt.

2. »Res facta «-Kompositionen, ${ }^{6}$ die sich auf eine präexistente Melodie einer polyphonen Chanson beziehen und einen neuen, textlosen Kontrapunkt dazu setzen.

3. Freie Neukompositionen, die kein Textincipit besitzen, häufig aber einen motto-ähnlichen Titel aufweisen, und die Banks in seinen ausführlichen Listen in Anlehnung an die frühen Solo-Lauten-Ricercare als »Consort Ricerare« bezeichnet. Es ist schwer, für diese Kompositionen einen geeigneten

4 J. Banks, Instrumental Consort (wie Anm. 2) - an dieser Stelle bin ich Nicole Schwindt zu Dank verpflichtet, die mich auf diese wichtige Neuerscheinung hingewiesen hat.

5 Warwick Edwards, »Songs Without Words by Josquin and his Contemporaries«, in: Music in Medieval and Early Modern Europe, hrsg. von Iain Fenlon, Cambridge 1981, S. 79-92.

6 Dieser Begriff wurde ebenfalls von Edwards (ebda., S. 82) auf Grundlage von Johannes Tinctoris' Verwendung des Terminus eingeführt. 
Genrebegriff zu finden und sie sind, obwohl sie sich von den anderen Gattungen abheben, schwer unter einer Bezeichnung zusammenzufassen.

Jedes dieser Genres analysiert er im Detail und stellt Repertoirelisten auf, in denen er die einzelnen Kategorien nach weiteren Aspekten der Kompositionen feiner unterteilt und klassifiziert.

Die Tatsache, dass dieses frühe >Instrumentalrepertoire nicht in Tabulaturen, sondern in Mensuralnotation überliefert ist, veranlasst Banks zu der Annahme, dass die Interpreten zum Kreis der ausgebildeten und gelehrten Sänger gehörten, zu denen auch die Komponisten dieser Stücke zählten und die mit der Notation aus ihrer täglichen Praxis heraus vertraut waren. Als Besetzung schließt er aufgrund von Ikonographie, Status des Instrumentes und vor allem aus dem für das Repertoire erforderlichen Ambitus auf ein Lautenensemble. Dabei geht er vom im 15. Jahrhundert üblichen Plektrumspiel der Laute aus und nimmt daher die einstimmige Verwendung des Instrumentes an, so dass die Aufführenden jeweils eine Stimme der vorliegenden Komposition ausführen konnten.

Er trennt diesen Kreis von auf der Laute gewissermaßen >nebenberuflich spielenden Sängern deutlich von den professionellen Instrumentalmusikern ab. Letztere sieht Banks als Virtuosen, die in erster Linie ohne Noten, improvisierend ihre Kunst präsentierten und in direkter Nachfolge der mittelalterlichen Menestrels stehen:

This is not the kind of music that we would expect of a medieval minstrel, whose performing world was essentially monophonic, improvisatory, soloistic and based on memorization rather than notation. Though it is impossible to be sure, the minstrel probably traded extensively on his instrumental virtuosity, and display for its own sake is not a feature of this music; though complex, the difficulties of Agricola's >Tandernaken< demand ensemble discipline rather than individual brilliance ...7

$\mathrm{Zu}$ dieser Sorte Interpreten würde man wohl die Musiker der professionellen Lauten-Duos des 15. Jahrhunderts rechnen, obgleich sie sich nicht der Einstimmigkeit verschrieben hatten, sondern auf improvisierte Diminutionen über einen gegebenen Tenor spezialisiert waren. Die Besetzung dieser Duos ist gut dokumentiert: ${ }^{8}$ in der Regel bestanden sie aus einer Laute und einer Quinterne (einem Oberstimmeninstrument der Lautenfamilie) oder

7 J. Banks, Instrumental Consort (wie Anm. 2), S. 40.

8 Siehe dazu vor allem Martin Kirnbauer, »Petrucci in the Fifteenth Century: The Lute Duos«, in: Venezia 1501. Petrucci e la stampa musicale. Atti del convegno internazionale Venezia ... 2001, hrsg. von Giulio Cattin und Patrizia Dalla Vecchia, Venedig 2005 (Edizioni Fondazione Levi, 3.B.6), S. 591-607, und Vladimir Ivanoff, »Das Lautenduo im 15. Jahrhundert «, in: Basler Jahrbuch für historische Musikpraxis 8 (1984), S. 147-162. 
aus zwei Lauten, wobei häufig eine größere und eine kleinere kombiniert wurden. Der Spieler des größeren Instruments hatte dabei die Funktion des $»$ Tenorista «, der das Fundament der Komposition lieferte - was neben schlichtem Halten der Tenorlinie auch eine Einrichtung von Tenor und Contratenor bedeutet haben könnte ${ }^{9}$-, während der andere Lautenist Diminutionen auf die Oberstimme extemporierte. Diese Oberstimmenimprovisationen konnten offenbar sehr virtuose Formen annehmen, wie zeitgenössische Beschreibungen festhalten. Besonders der Lautenist Pietrobono muss mit solchem Spiel seine Zeitgenossen begeistert haben. Aurelio Brandolini beschreibt in seinen Lobversen auf den Lautenisten dessen Spiel:

Staune gebannt, wie seine linke Hand die gesamte Laute entlangläuft, wie seine Hand schnell über die klangvollen Saiten gleitet. Du wirst bewundern, wie alle seine Finger gleichzeitig fliegen, wie eine Hand an so vielen Orten zugleich sein kann. Bald stürmt sie in die höchsten Lagen, bald läuft sie bis zu den tiefsten Tönen. ... Und es gibt keine Hoffnung auf Rast, weder für Plektrum noch für Finger gibt es eine Pause, bis das Lied beendet ist. ${ }^{10}$

Leider sind Beispiele dieser Ensemblepraxis so gut wie nicht überliefert, was vor allem bei der improvisierten Oberstimme gewissermaßen in der Natur der Sache liegt. Die einzigen Überlieferungen, die einigermaßen an die Beschreibungen herankommen könnten, sind die sechs Intavolierungen für zwei Lauten in Francesco Spinacinos Tabulaturen von 1507. ${ }^{11}$

Sie wirken in vielerlei Hinsicht außergewöhnlich und unterscheiden sich deutlich von anderen verzierten Kompositionen dieser Zeit, die sich normalerweise auch bei Diminutionen im Rahmen traditioneller kontrapunktischer Techniken bewegen. Während bei Spinacino die Tenor-Laute die Unterstimmen der vorgegebenen Kompositionen in rhythmisch vereinfachter Form und stetig pulsierendem Rhythmus präsentiert, scheint der primäre Zweck der diminuierten Oberstimme darin zu liegen, den vollen Tonumfang der Laute auszunutzen und in konstanter Bewegung zu bleiben. Kadenzen des

9 Fallows wies bereits darauf hin, dass der Tenorista im Lautenduo wahrscheinlich die beiden Unterstimmen dreistimmiger Chansons für sein Instrument zusammenlegte. (David Fallows, $\gg 15^{\text {th }}$-century Tablatures for Plucked Instruments: A Summary, a Revision and a Suggestion«, in: The Lute Society Journal 19 (1977), S. 7-33.

10 Aurelio Brandolini, Libellus de Landibus musicae et Petroboni (Ms., 1473), Kap. 3, Verse 79-84 und 99f.; übersetzt nach dem Zitat bei F. Alberto Gallo, Music in the Castle. Troubadours, Books, and Orators in Italian Courts of the Thirteenth, Fourteenth, and Fifteenth Centuries, Chicago 1995, S. 123f.

11 Francesco Spinacino, Intabulatura de lauto. Libro primo, Venedig: Ottaviano Petrucci 1507, RISM $1507^{5}$, fol. $12^{\mathrm{r}}-25^{\mathrm{v}}$, und [...] Libro secondo [...] RISM $1507^{6}$, fol. $38^{\mathrm{v}}-41^{\mathrm{r}}$, Reprint hrsg. von François Lesure, Genf 1978. 
Originals werden zwar in der Regel beachtet, ansonsten entfernt sich die diminuierende Stimme aber sehr weit vom Original und gewinnt eine Eigendynamik in Skalenläufen und kleinen Figuren, die sich über eine Harmonie mit den Unterstimmen oft hinwegsetzt. Der Verschriftlichungsprozess dieser eigentlich schriftlosen Kunst kann nicht ohne Verluste vonstatten gegangen sein. Ohne sie folglich allzu wörtlich zu nehmen, können sie dennoch einen prinzipiellen Eindruck davon geben, wie Bearbeitungen im Lautenduo des 15. Jahrhunderts ausgesehen haben. ${ }^{12}$

Somit unterscheidet sich sowohl das Repertoire als auch sein Interpretenkreis deutlich vom Lautenensemble, das Banks für sein »Consort Repertory« annimmt. Allein die Tatsache, dass eine Niederschrift für diese Kompositionen erfolgte, wäre damit ein weiterer Hinweis auf die Besonderheit des Repertoires in einem Umfeld, in dem instrumentales Repertoire generell individuell und schriftlos war.

Die Instrumentierung dieser Kompositionen mit Violen- und anderen Consorts, die sich für das Repertoire ebenfalls hervorragend eignen, war gemäß Banks erst ein zweiter Schritt, der auf die Etablierung des Repertoires für Lautenensemble folgte. Das Lautenensemble bereitete gewissermaßen anderen homogenen Instrumentenensembles, für die dann schließlich eigene Repertoires geschaffen wurden, den Weg. ${ }^{13}$

So rund die Argumentation wirkt, so plausibel das Ergebnis klingt und so schmeichelhaft es für einen Lautenisten auch erscheinen mag, ein gesamtes, frühes Lautenrepertoire zugeschrieben zu bekommen, so bleibt man doch gewahr, dass dieses Argumentationsgebäude Banks' letztlich auf Indizienbeweisen fußt.

Ein Eckpfeiler seiner Beweisführung ist die angenommene, einstimmige Verwendung der Laute im 15. Jahrhundert als Plektruminstrument, die es ermöglicht, dass einzelne Linien mit hohem Ambitus in einem komplexen polyphonen Gerüst auch von >guten Laien< ausgeführt werden können.

Die vielen Abbildungen dieser Zeit zeigen, was die Anschlagstechnik angeht, zwar, dass die Laute im 15. Jahrhundert noch vornehmlich mit dem Plektrum gespielt wurde - auch zahlreiche Nennungen von Plektrumlautenisten, darunter besagter Pietrobono, belegen den Augenschein. Erst zum Ende des Jahrhunderts häufen sich die Darstellungen merklich, die ein Fingerspiel zeigen, bis schließlich Anfang des 16. Jahrhunderts das Fingerspiel laut Ikonographie überwiegt. Dabei kann kein radikaler Wechsel von

12 Siehe dazu M. Kirnbauer, Petrucci (wie Anm. 8), S. 602f.

13 Siehe dazu J. Banks, Instrumental Consort (wie Anm. 2), S. 35. 
einer zur anderen Spielweise festgestellt werden, denn auch das Plektrumspiel hielt sich noch bis weit ins 16. Jahrhundert. ${ }^{14}$

Eine Annahme, die man seit Beginn der wissenschaftlichen Beschäftigung mit mittelalterlichem Lautenspiel bis hin zu Banks' Monografie fast ohne Widerspruch immer wieder antrifft, ist aber, dass Plektrumspiel auf der Laute mit einstimmigem Spiel gleichzusetzen sei. Von der Anreißtechnik kann jedoch nicht auf ein- oder mehrstimmiges Spiel geschlossen werden, denn entgegen der verbreiteten Meinung, dass die Plektrumlaute allein für monophone Linien verwandt wurde und erst die mit Fingern gezupfte Laute Mehrstimmigkeit auf dem Instrument gestattete, ist es sehr wohl möglich, polyphon - und dabei auch virtuos - auf der Plektrumlaute zu spielen. ${ }^{15}$ Der

14 Noch 1523 findet sich die Erwähnung von Plektrumspiel im Lautenensemble. Siehe J. Banks, Instrumental Consort (wie Anm. 2), S. 46.

15 So wird zum Beispiel bei der Erwägung von Besetzungen für die Intavolierungen im Codex Faenza Folgendes festgestellt: »Let us consider first the possibility of performing both parts on a single instrument. Lute can immediately be eliminated because although it possessed the necessary range, it was not played polyphonically before the late $15^{\text {th }}$ century. Until then a plectrum was used, precluding the performance of more than one line « (Timothy J. McGee, »Instruments and the Faenza Codex«, in: Early Music 14, 1986, S. 482). Zwar wird polyphones Lautenspiel von McGee für das Repertoire im späten 15. Jahrhundert in Aussicht gestellt, aber allein unter der Vorgabe, dass dann bereits mit Fingern an Stelle des Plektrums gespielt wurde. Eine solistische Ausführung von Intavolierungen im Stile des Codex Faenza mit der Plektrumlaute ist aber auf jeden Fall möglich. Eventuell würde ein Interpret einzelne Stellen verändern, seiner individuellen Technik anpassen oder vereinfachen wollen, im Prinzip stellt die Mehrstimmigkeit solcher Quellen für den versierten Plektrumspieler aber kein Hindernis dar. Ob diese Musik auch im 15. Jahrhundert solistisch von Plektrumspielern aufgeführt wurde, kann nicht mit Sicherheit gesagt werden, einen physikalischen Hinderungsgrund kann man aber nicht annehmen. Weitere Beispiele in der Sekundärliteratur finden sich bei Roland Eberlein, »The Faenza Codex: music for organ or for lute duet? «, in: Early Music 20 (1992), S. 461f., wo er die Thesen McGees zu widerlegen sucht, die Annahme McGees über das einstimmige Plektrumspiel aber wiederholt und bestärkt: »... lutes were not played polyphonically before the late 15th century«, ferner Howard Mayer Brown, »Instruments«, in: Performance Practice. Music Before 1600, hrsg. von dems. und Stanley Sadie, New York 1989, S. 175: »The new technique [mit Fingern] enabled performers to play polyphonic music on their instruments", und Keith Polk (German Instrumental Music of the Late Middle Ages. Players, Patrons and Performance Practice, Cambridge 1992, S. 218f., Anm. 42), der ebenfalls von einstimmigem Plektrumspiel ausgeht, findet die Vorstellung, dass ein Plektrumspieler auch Polyphonie umsetzen könnte, immer noch »intriguing«. Obwohl Crawford Young die Möglichkeit mehrstimmigen Spiels mit der Plektrumlaute aus der Erfahrung eines Lautenisten heraus bespricht (»Lute, Gittern, \& Citole«, in: A Performer's Guide to Medieval Music, hrsg. von Ross W. Duffin, Bloomington 2000, S. 363), geht auch Banks (Instrumental Consort, wie Anm. 2, S. 32) noch von der oben erwähnten Gleichsetzung aus: »The fact that fifteenth-century lutenists had been essentially plectrum players shows that 
Einsatz von Bünden ab 1400 wurde in der Vergangenheit bereits als Argument für das mehrstimmige Spiel auf der Plektrumlaute angeführt. ${ }^{16}$ Dem ungeachtet kann die Wahl der Anschlagstechnik ebenso wie die Entscheidung für den Einsatz von Bünden durchaus andere Gründe gehabt haben. Beispielsweise könnten eine veränderte Klangästhetik ${ }^{17}$ oder persönliche Spielpräferenzen des jeweiligen Musikers ausschlaggebend gewesen sein, so dass diese Faktoren nicht notwendigerweise die ein- oder mehrstimmige Verwendung des Instrumentes belegen.

Es werden im 15. und frühen 16. Jahrhundert zwar Lautenensembles mit bis zu vier Spielern erwähnt, ${ }^{18}$ die offenbar Plektren benutzten und dann wahrscheinlich Einzellinien übernahmen, und auch die Improvisationskunst Pietrobonos über einen Tenor wird einstimmig vonstatten gegangen sein, aber schon die Aufgabe des ihn begleitenden Tenorista könnte hier vielfältiger gewesen sein, als seine Funktionsbezeichnung anzudeuten scheint: Die oben erwähnte Möglichkeit der Intavolierung und Zusammenlegung von Tenor und Contratenor in ein Instrument ist sehr naheliegend, vor allem wenn der Contratenor eher füllende Funktion hat.

Die einzigen überlieferten Tabulaturen, die sich auf die Praxis des professionellen Lautenensembles im Stile Pietrobonos beziehen könnten - die besagten sechs Lautenduos in den Tabulaturdrucken von Spinacino - bestätigen dieses Vorgehen: ${ }^{19}$ Für die begleitende >Tenorlaute< wurden die Unterstimmen der zugrunde liegenden Chansonsätze (in einem Fall einer untextierten Komposition) in ein Instrument zusammengelegt, während die Oberstimme sich in freien, diminuierenden Läufen darüber einstimmig ergeht. Zwar hat man zur Zeit von Spinacinos Druck die Laute bereits auch mit Fingern gespielt, aber diese Lautenduos weisen in ihrer Charakteristik eindeutig auf eine Praxis des 15. Jahrhunderts zurück - und wie oben bereits dargelegt, bereiten die Unterstimmenintavolierungen von Spinacino für einen Plektrumspieler keine Probleme.

it was essentially a monophonic rather than polyphonic instrument, in other words one that played single lines of the type found with such large ranges here.«

16 Siehe V. Ivanoff, Das Lautenduo (wie Anm. 8), S. 154.

17 Es gibt Hinweise darauf, dass die Bünde der Laute eventuell dazu eingesetzt wurden, einen »Schnarrklang « zu erzeugen, wie er bei der Harfe im 15. und 16. Jahrhundert üblich war. Da dieses Feld aber bislang weder ausgiebig recherchiert noch erprobt wurde, muss hier weitere Forschung für klarere Aussagen abgewartet werden.

18 Siehe dazu das Kapitel »The lute consort«, in: J. Banks, Instrumental Consort (wie Anm. 2), S. 36-40.

19 Siehe dazu M. Kirnbauer, Petrucci (wie Anm. 8). 
Dass sich die Plektrumlaute auch für das Spiel einzelner Linien eignet, ist unumstritten und bietet sich dabei besonders für die Umsetzung diminuierter Stimmen an. Es trifft sicher ferner zu, dass das Plektrumspiel ursprünglich einer tendenziell einstimmigen Tradition entstammt, während das spätere Fingerspiel eher vertikale Strukturen begünstigt. Beide Techniken und Anwendungsbereiche stellen aber Extreme dar, die an den Endpunkten einer Entwicklung stehen - dazwischen erstreckt sich ein langer Zeitraum, der einstimmiges Spiel mit Fingern ebenso plausibel erscheinen lässt wie Mehrstimmigkeit mit Plektrum. Und somit widerlegt diese Feststellung auch nicht die Argumentation von Banks zugunsten eines Lautenensembles mit monophon spielenden Interpreten, erweitert aber die Möglichkeiten des Lauteneinsatzes gewaltig: Spieltechniken und Prinzipien der Umsetzung auf der Laute sind für diese Epoche nicht auf nur eine Praxis reduzierbar.

Diese Fülle an Möglichkeiten birgt ein großes Potenzial: Es steht uns bei der Ausführung von Musik im späten 15. Jahrhundert auf der Laute eine Vielfalt an Techniken zur Verfügung, mit denen wir experimentieren und sie in Aufführungen verwenden können.

Ziel des Beitrags ist es daher nicht, eine >richtige< Besetzung für die textlosen Kompositionen Agricolas zu finden, sondern Wege aufzuzeigen, wie ein Lautenist das vorliegende Repertoire, gleich ob textiert oder untextiert, sinnvoll auf der Laute realisieren kann. ${ }^{20}$

\section{Einsatz der Laute als einstimmiges Ensembleinstrument}

Eine Möglichkeit für die Instrumentierung wäre der Einsatz einer oder mehrerer einstimmig gespielter Lauten innerhalb eines klanglich homogenen Ensembles, also einer Besetzung, die aus Instrumenten ähnlicher, wenn nicht gar gleicher Tonerzeugung besteht.

Zunächst käme dafür das von Banks postulierte Lautenensemble in Frage, das als Konstellation belegt ist und sinnvolle Ergebnisse verspricht. Bei Besetzung aller Stimmen mit Lauten, also mit einem sehr hohen Grad an Klangverschmelzung, kann es aber mitunter schwer werden, einzelnen Stimmführungen zu folgen. Je nachdem welche Charakteristika der Komposition oder der Instrumente die Aufführenden hervorheben möchten, kann dieses Ergebnis entweder gewünscht sein, oder sich durch Bearbeitung der Einzel-

20 Dabei stütze ich mich neben eigenen vor allem auf die langjährigen Erfahrungen, die an der Mittelalterabteilung der Schola Cantorum Basiliensis und im Besonderen von Crawford Young zu diesem Thema gesammelt wurden. 
linien beheben lassen - beispielsweise durch den Einsatz von Verzierungen und Tonaufspaltungen, kurzum durch instrumentengerechte Arrangements.

Ein typischer Fall für die Notwendigkeit instrumentenspezifischer Eingriffe sind die in diesen Kompositionen häufig auftretenden langen Töne: Bei Besetzung mit nicht-gestrichenen Saiteninstrumenten sind lang ausgehaltene Töne schwer darstellbar, weil der angeschlagene oder gezupfte Ton schnell verklingt. Dadurch wird einerseits die betreffende Note als Kontrapunkt zu eventuell gleichzeitig erklingenden, bewegteren Stimmen weniger gut wahrgenommen, andererseits verliert der Hörer leicht den linearen $\mathrm{Zu}$ sammenhang einer Stimme.

Diese Beobachtungen müssen nicht zwingend in die Schlussfolgerung münden, eine Komposition oder eine Stimme innerhalb einer Komposition, die solche langen Töne enthält, sei generell ungeeignet für das Spiel auf der Laute; noch müssen sie zu der Feststellung führen, diese Stimme wäre für eine explizite Besetzung geschrieben worden. Für den Zupfer gibt es die Möglichkeit der Tonwiederholung, die auch praktisch angewandt wurde, wie man am Beispiel der Unterstimmenintavolierungen der Spinacino-Duos sehen kann (siehe Notenbeispiel 1).

Ferner ist ein Auffüllen durch Verzierungen oder kleine Figuren möglich. Je weiter man diese instrumententypischen Bearbeitungen treibt, in die sicherlich auch die Erfahrungen und technischen Fertigkeiten des jeweiligen Spielers eingehen können und zum Teil von erfahrenen Spielern ohne große Überlegungen automatisch vorgenommen werden, desto näher gerät man dabei in den Bereich der wirklichen Instrumentalbearbeitung, der Intavolierung.

Weitere Lösungen für einen zu gleichartigen Klang der einzelnen Stimmen wären Experimente mit Tonfärbungen auf dem Instrument oder die Besetzung mit Instrumenten ähnlicher, aber nicht identischer Tonerzeugung. ${ }^{21}$ Diese Parameter sind mit den oben genannten Ideen $\mathrm{zu}$ instrumentenspezifischen Bearbeitungen frei kombinierbar.

Notenbeispiel 1 (auf der folgenden Seite): anonym, »J'ay pris amour «(Anfang), oben: Lautenversion nach Spinacino 1507, unten: Vorlage nach dem Chansonnier Nivelle de la Chaussée, F-Pn, Rés. Vmc 57, fol. $71^{v}-72^{r}$

21 Ein solcher Besetzungsversuch am Beispiel von Agricolas Pater meus agricola est mit Chekker (einem frühen Tastenintrument), Dulcemelos (einer Form des mittelalterlichen Hackbretts) und Quinterne zeigte: Der Chekker mit mechanisch angezupften Metallsaiten, das Dulcemelos mit durch Klöppel angeschlagenen Metallsaiten und die Quinterne mit plektrumgezupften Darmsaiten sind sich im Klang ähnlich genug, um als homogen wahrgenommen zu werden, die Klangerzeugung ist aber noch ausreichend unterschiedlich, um einzelne Stimmführungen deutlicher hervorzuheben. 

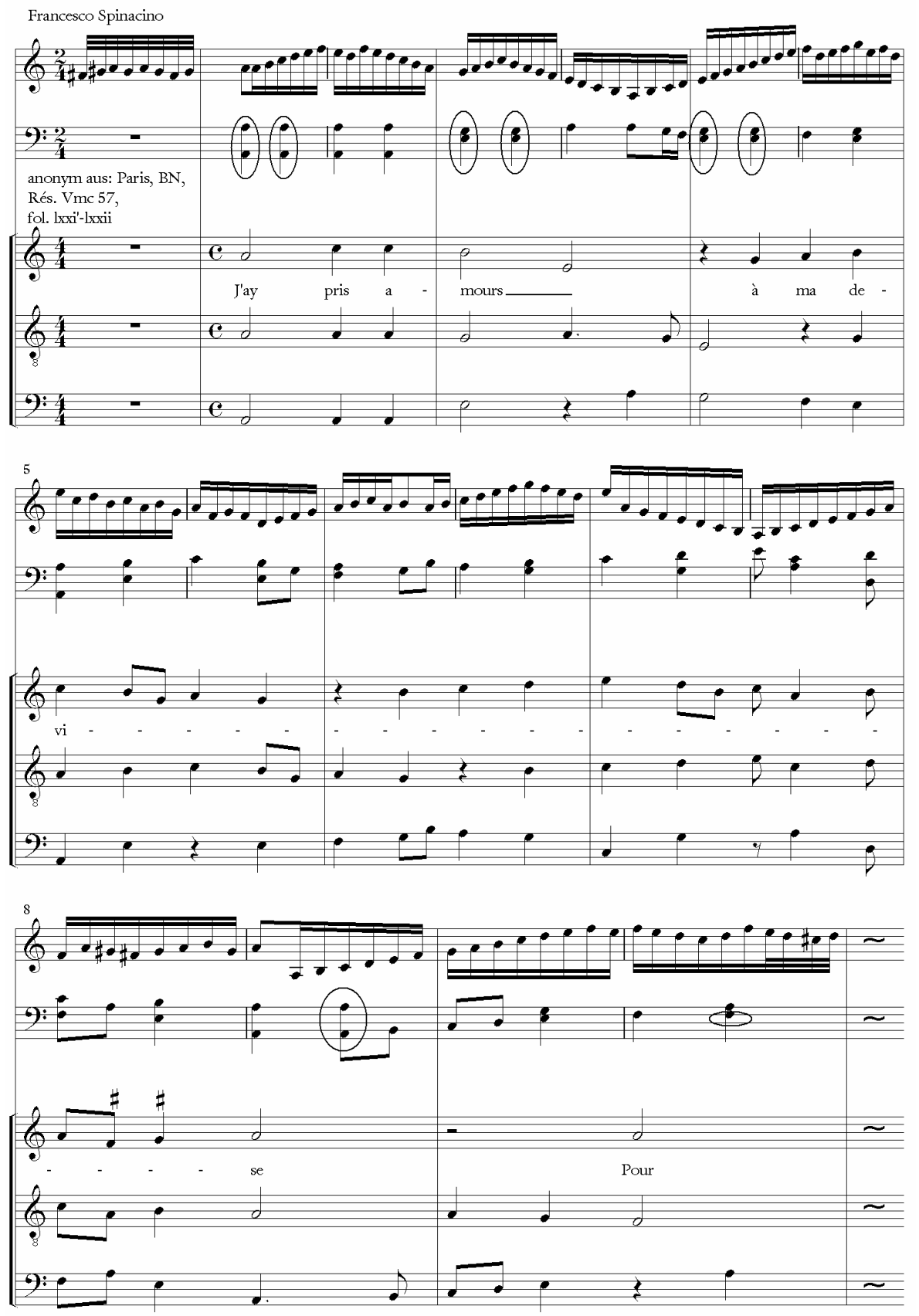
Außerdem besteht natürlich die Möglichkeit, eine Laute in einem gemischten Ensemble einstimmig zu spielen - wobei »gemischt« hier eine Besetzung meint, die Instrumente verschiedener Klangerzeugung und vor allem Klangfarbe vereint, beispielsweise Bläser, Streicher und Zupfer - aber natürlich auch Sänger. In einer solchen Besetzung bietet es sich an, die Laute mit einem Plektrum zu spielen, was sie perkussiver und durchsetzungsfähiger macht - gerade bei kräftigem Streicherklang oder Einsatz einer Harfe mit Schnarrhaken kann dies gewährleisten, dass die Laute akustisch nicht >untergeht $<$. Trotz dieser Maßnahme wird aber eine einstimmig geführte Laute gegenüber zum Beispiel Streichern in der Regel nicht als gleichberechtigt, sondern hierarchisch untergeordnet wahrgenommen. Was bei einem burgundischen Kantilenensatz noch gut funktioniert, wo Stimmfunktionen klarer getrennt sind, kann bei manchen Kompositionen aus Agricolas Generation zu einer ungewollten Betonung einer Stimme auf Kosten anderer führen. ${ }^{22}$

Von daher gilt das übliche Verfahren, die Wahl der Besetzung eng an der Satzkonstruktion der gewählten Komposition zu orientieren, für dieses Repertoire in besonderem Maße. Der Tenor des De tous biens plaine $\mathrm{II}^{23}$ etwa (siehe Notenbeispiel 2) ließe sich passend mit einem Instrument besetzen, das liegende Töne für den gedehnten Cantus firmus zur Verfügung stellen kann, während die rastlosen Außenstimmen gut von Zupfern gespielt werden können. Gleiches gilt für das zweistimmige Gaudeamus omnes in Domino (siehe Notenbeispiel 3) mit ruhigem Tenor und bewegter Oberstimme, das in gemischten Besetzungen gut funktionieren kann. ${ }^{24}$

22 Eine experimentelle Besetzung für Agricolas Cecus non judicat de coloribus im gemischten Ensemble (in diesem Fall besetzt mit Dulcemelos, Blockflöte und Laute) führte diesen Aspekt vor Augen: Als Ausführende stellten wir fest, dass wir den Satz sehr wohl mit gleichberechtigten Stimmen hörten und deren Interaktion nachvollziehen konnten. Die Wahrnehmung Außenstehender aber, beispielsweise beim Vorspiel oder im Konzert, war eine deutlich andere: durch den Einsatz der Flöte im Cantus wurde die Komposition als Oberstimmensatz wahrgenommen, begleitet durch zwei Saiteninstrumente in den Unterstimmen.

$23 \mathrm{Zu}$ allen Sätzen siehe die Ausgabe Alexandri Agricola Opera omnia, hrsg. von Edward R. Lerner, Bd. 5: Cantiones, Musica Instrumentalis, Opera dubia, American Institute of Musicocogy 1970 (Corpus Mensurabilis Musicae, 22.5).

24 Bei diesen konkreten Besetzungsvorschlägen handelt es sich lediglich um Ideen, die im Experiment ausprobiert wurden und zu den genannten Beobachtungen führten. Sie erheben keinen Anspruch auf Absolutheit - ein Hörer des 15. Jahrhunderts mag die formulierten Zusammenhänge ganz anders wahrgenommen haben. 


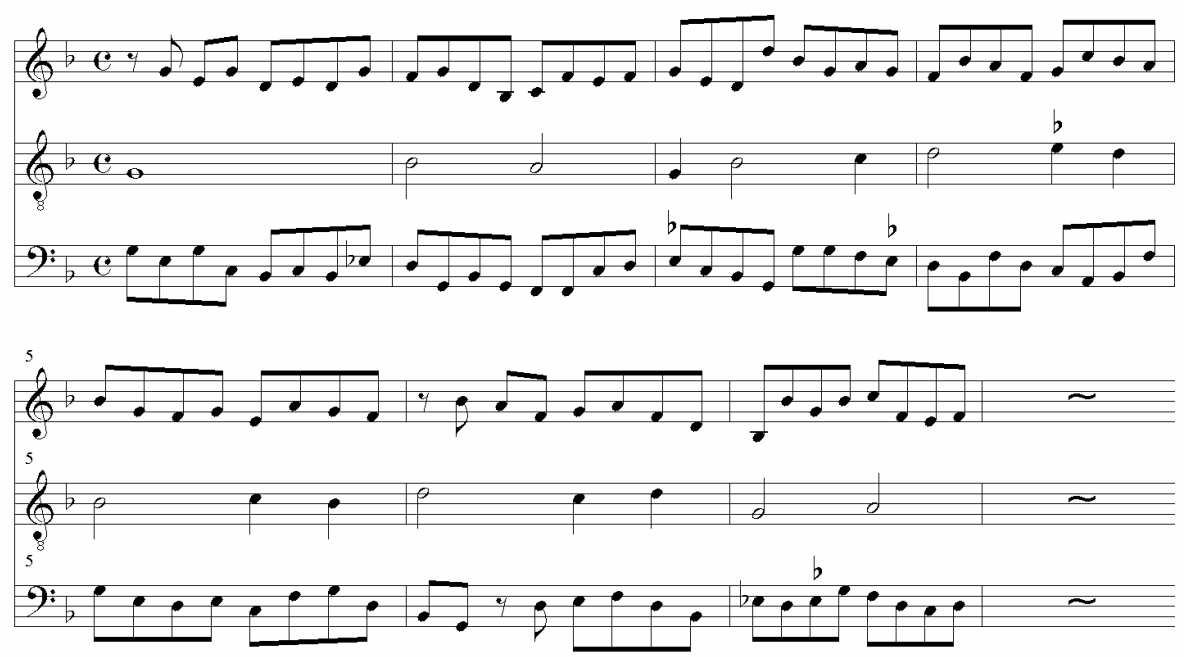

Notenbeispiel 2: A. Agricola, De tous biens plaine II (Anfang)
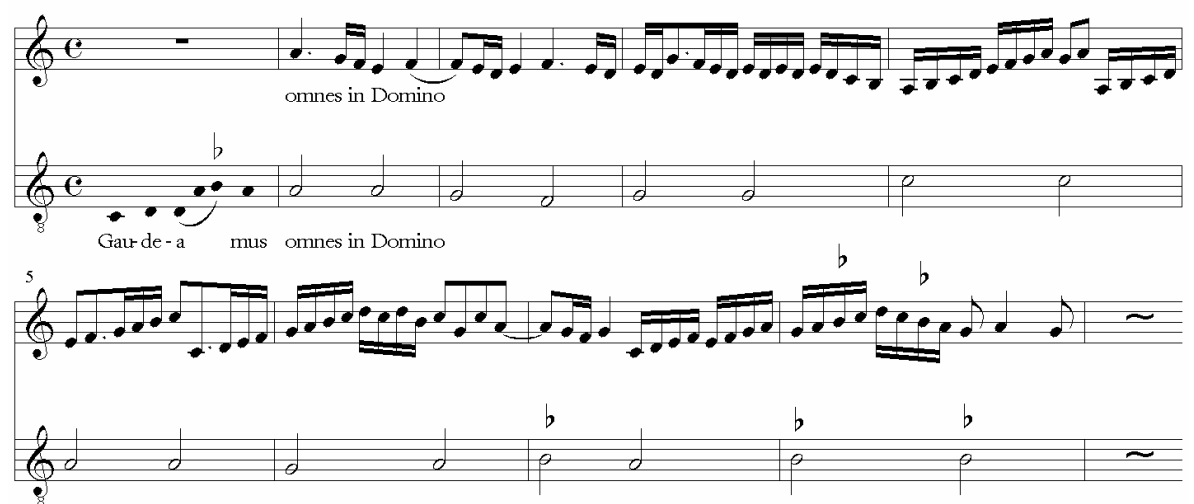

Notenbeispiel 3: A. Agricola, Gaudeamus omnes in Domino (Anfang)

\section{Einsatz der Laute als Soloinstrument}

Eine weitere, recht offensichtliche Möglichkeit für den Einsatz der Laute ist die Solobearbeitung. Mit dem Beginn der Überlieferung von Lautentabulaturen im späten 15. Jahrhundert haben wir einen klaren Anhaltspunkt für einen zeitgenössischen Umgang mit der Laute. 
Dabei sind die überlieferten Tabulaturen freilich nicht mehr als ein Echo auf eine Praxis der schriftlosen Lautenbearbeitung, die vor allem von professionellen Spielern angewandt wurde und ihren Weg in die Schriftlichkeit nur in Ausnahmefällen fand. Die Verbreitung der Lautentabulatur hängt vermutlich stark mit einem Bedarf seitens wohlhabender, dilettierender Lautenisten zusammen - wobei »dilettierend « im besten Sinne des Wortes zu verstehen ist und im Gegensatz zu den professionellen Spielern steht, die auf Tabulaturen generell nicht angewiesen waren. Von daher können diese Fassungen nur Hinweise geben, wie Kompositionen in Lautenbearbeitungen umgewandelt wurden, denn sie wurden ja in erster Linie für Laien hergestellt und nicht immer von Fachleuten. Ferner kann man davon ausgehen, dass nicht jeder Berufslautenist seine persönlichen Bearbeitungen einer breiten Öffentlichkeit preisgeben wollte, dass eventuell Veränderungen oder Vereinfachungen für die Drucklegung vorgenommen wurden und in vielen Fällen auch die Notationsmethoden zur Darstellung des eigentlich Gemeinten nicht ausgereicht haben werden - was auch die oft fehlenden rhythmischen Zeichen der frühen Tabulaturhandschriften erklären könnte. ${ }^{25}$

In drei frühen Quellen des 16. Jahrhunderts finden sich Lautenintavolierungen von Stücken Alexander Agricolas. Bemerkenswert ist dabei, dass sich fast alle Genres unter den Bearbeitungen finden - neben textlosen Vorbildern auch Chansons und Motetten -, so dass sich keine Bevorzugung eines Genres gegenüber einem anderen für die Lautenbearbeitung erkennen lässt.

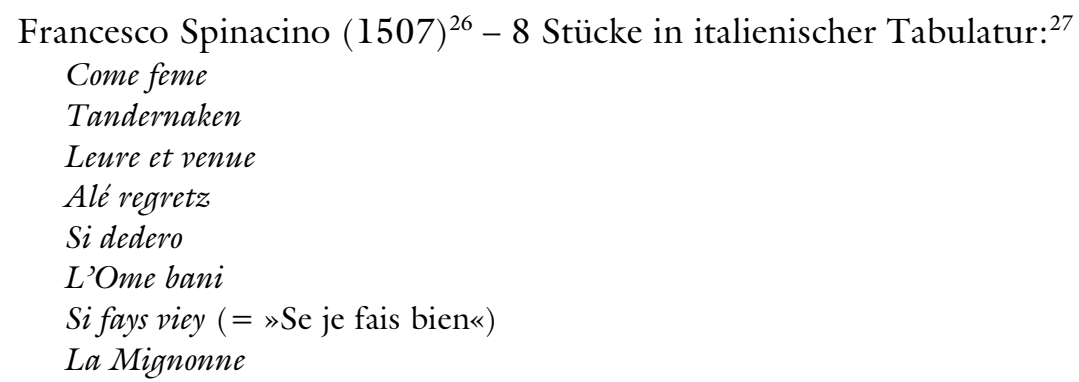
nach ihrer Abfolge in den Quellen sortiert.

27 Alle Tabulaturen sind in den Drucken Spinacinos ohne Autorangaben wiedergegeben. 
Vincenzo Capirola $(1517)^{28}-2$ Stücke in italienischer Tabulatur: ${ }^{29}$

Oblivier (= Oublier vueil tristesse)

Si dedero

Hans Neusidler $(1536 a)^{30}-1$ Stück in deutscher Tabulatur:

Alexander agricola (= Cecus non judicat de coloribus)

[Ander nacken up dem Rbin $]^{31}$

Hans Neusidler $(1536 b)^{32}-3$ Stücke in deutscher Tabulatur:

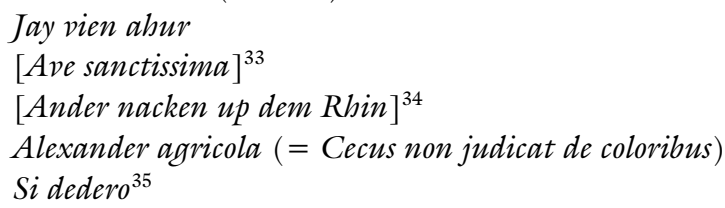

Die drei vorliegenden Tabulatursammlungen entstammen völlig unterschiedlichen Zusammenhängen und wurden für sehr verschiedene Zwecke geschaffen:

Im ersten überlieferten Tabulaturdruck, den beiden Lautenbüchern des Francesco Spinacino, die 1507 bei Petrucci in Venedig verlegt wurden, finden sich acht Bearbeitungen von Kompositionen Agricolas. Betrachtet man unter diesen Fassungen zum Beispiel das Alé regretz genauer, so wird deutlich, dass diese Intavolierung einen Kundenkreis von interessierten Laien überfordert haben dürfte. Spinacino wählte bei seiner Bearbeitung auf der Laute eine sehr hohe Transposition, die den Spieler ohne Not in extreme Lagen zwingt: weder der Ambitus noch die Darstellbarkeit des Kontrapunktes im Fingersatz erfordern diese Transposition. Setzt man das Stück tiefer, so ergeben sich für den Spieler wesentlich angenehmere Positionen, ohne dass die transparente Stimmführung zu leiden hätte. Ähnliches gilt für

28 US-Cn, Case MS VM C.25. Die Handschrift mit dem Titel Compositione di meser Vincenzo Capirola, gentil homo bresano wurde etwa 1517 von einem Schüler Capirolas namens Vidal hergestellt, Reprint-Ausgabe Florenz 1981 (Archivum musicum. Collana di testi rari, 39).

29 Auch in der Capirola-Handschrift finden sich generell keine Autorangaben.

30 Hans Neusidler, Ein newgeordent künstlich Lautenbuch in zwen Theyl getheylt: der erst ..., Nürnberg: Johann Petreius 1536 (RISM 1536 ${ }^{12}$ ). Reprint-Ausgaben Neuss 1974 (Institutio pro arte testudinis, A.1) und Stuttgart 2004 (Faksimile-Edition Laute, 6).

31 Implizit über die Zuordnung in 1536b fälschlicherweise Agricola zugeschrieben.

32 Hans Neusidler, Der ander Theil des Lautenbuchs, Nürnberg: Johann Petreius 1536 (RISM $1536^{13}$ ), Reprint-Ausgabe Neuss 1974 (Institutio pro arte testudinis, A.3). In den Drucken Neusidlers, besonders in 1536b, wird die Autorzuordnung fast zum Prinzip erhoben.

33 Vermutlich fälschlicherweise Agricola zugeschrieben.

34 Vermutlich fälschlicherweise Agricola zugeschrieben.

35 Von Neusidler fälschlicherweise Jacob Obrecht zugeschrieben. 
die Tandernaken-Bearbeitung, ein Stück, das schon im Ensemblespiel eine Herausforderung darstellt: die Solo-Lautenfassung verlangt dem Spieler Enormes ab - Aufwand und Effekt halten sich hier kaum die Waage.

Auch die Fingersätze, die Spinacino sowohl in seinen Intavolierungen für Sololaute wie auch in den Oberstimmen seiner Duos vorgibt, wirken zum Teil recht exzentrisch - man fragt sich oft, warum er bestimmte Figuren nicht günstiger legte. Der Unterstützung von Stimmführungen sind diese Entscheidungen zumeist jedenfalls nicht geschuldet. Abgesehen davon, dass Fingersätze sehr von der individuellen Technik und den Gewohnheiten des jeweiligen Spielers abhängig sind, erscheinen Spinacinos Sätze dennoch außergewöhnlich.

Von daher stellt sich die Frage, wie diese Notentexte entstanden, und wie wörtlich sie zu nehmen sind.

Möglicherweise wurden die Intavolierungen relativ kurzfristig erstellt, da Petrucci unter erheblichem Druck stand, eine Tabulatur herauszugeben, um sein bis dahin nicht wahrgenommenes und inzwischen angefochtenes Privileg zu verteidigen. ${ }^{36}$ Eventuell wurden die Bearbeitungen dann in Ermangelung eines geübten Intavolierers unter Zeitdruck von jemandem vorgenommen, der es lediglich verstand, ein Notationssystem in ein anderes zu übertragen - eine mensural notierte Vorlage in eine Tabulatur -, der aber vielleicht selbst keine große Praxis im Lautenspiel besaß. Die Folge wären Tabulaturen, die eher mechanisch als aus musikalischer Erfahrung heraus entstanden. ${ }^{37}$ In ihrer Anlage gleichen diese Drucke sehr den textlosen Chansonniers, die Petrucci ebenfalls herausgegeben hatte. Möglicherweise sind sie nach ähnlichem Muster entstanden und sollten wohl auch, wie die Chansonniers, berühmte Kompositionen enthalten, um für gute Verkaufszahlen zu sorgen. ${ }^{38}$

36 Siehe dazu in der Einleitung zur Edition: Compositione di Meser Vincenzo Capirola. LuteBook (circa 1517) hrsg. von Otto Gombosi, Neuilly-sur-Seine 1955 (Publications de la Société de Musique d'autrefois. Textes musicaux, 1), S. XXIX.

37 An dieser Stelle möchte ich mich herzlich bei Martin Kirnbauer bedanken, der einige dieser Ideen im Gespräch vorbrachte.

38 Banks meint in diesem Zusammenhang: »In terms of repertory and layout, the Spinacino tablatures are essentially textless chansonniers «, und stellt die Tabulaturen in dieser Hinsicht in eine Reihe mit Odhecaton, Canti B, Canti C, aber auch solchen handschriftlichen Quellen wie dem Basevi-Codex (J. Banks, Instrumental Consort, wie Anm. 2, S. 31). Und Gombosi stellt fest: »Of the 54 transcriptions contained in Spinacino's two books not less than 44 are taken from them [Petruccis vorangegangenen Drucken], mostly from the Odhecaton (29) and Canti C (10)«(O. Gombosi, Compositione, wie Anm. 32, S. XXIX). 
Capirolas Handschrift hingegen war offensichtlich nicht für den kommerziellen Markt gedacht: Während die Intavolierungen von Spinacino noch viele Fragen aufwerfen, mitunter, weil über seine Person außerhalb der beiden Drucke praktisch nichts bekannt ist, so entstammen die ca. zehn Jahre später, gegen 1517 niedergeschriebenen Tabulaturen des Vincenzo Capirola, unter denen sich auch zwei Agricola-Kompositionen befinden, nachweisbar einer lautenistischen Praxis und dem kreativen Spannungsfeld zwischen einem etablierten Virtuosen und Lehrer und seinen Schülern. ${ }^{39}$ Einer von ihnen legte, wie das Vorwort verrät, die Kompositionen und Intavolierungen seines Mentors in dieser prachtvollen Tabulaturhandschrift nieder. ${ }^{40}$ Auch die Art, wie die Vorlagen für das Spiel auf der Laute umgesetzt wurden, lässt intensive Beschäftigung mit dem Instrument und idiomatische Wendungen erkennen - die Lage ist in der Regel nicht so hoch gewählt wie bei Spinacino, und der spieltechnische Anspruch liegt eher im Bereich instrumentenspezifischer Virtuosität als im $>$ Kampf $\prec$ mit Lage und Transposition. Zwar sind diese Aspekte absolut gesehen sehr subjektiv und würden von Spieler zu Spieler unterschiedlich beurteilt werden, im relativen Vergleich mit den Tabulaturen Spinacinos lassen sich die erwähnten Beobachtungen aber zumindest als Tendenzen feststellen.

Die dritte Tabulatursammlung, die einzelne Stücke Agricolas enthält, sind die beiden Bände des 1536 im Druck erschienenen Newgeordent künstlich Lautenbuch des Hans Neusidler. Schon aus den Untertiteln zu den Bänden ist ersichtlich, dass die Anlage dieser zwei zusammenhängenden Drucke einem didaktischen Gesamtkonzept unterworfen ist: Der erst [Teil] für die anfahenden Schuler / die aus rechter kunst vnd grundt nach der Tabulatur / sich one einichen Meyster darin zü̈ben haben..${ }^{41}$ und Der ander theil des Lautenbichs. Darin sind begriffen / vil außerlesner kunstreycher stuck ... für die geübten vnnd erfarnen diser kunst ... ${ }^{42}$

Während bei Spinacino die Beweggründe für den Tabulaturdruck noch vage bleiben, lässt Neusidler durch ausführliche Vorworte und Beischriften

39 »Intabulated vocal compositions in Petrucci's prints [für die Laute] are limited by considerations for the dilettante. ... Capirola goes far beyond the standards of Spinacino« (O. Gombosi, ebda.).

$40 \gg \mathrm{I}$, Vitale, considering that many divine works have been lost through ignorance of their owners, and wishing that this quasi divine book I have written should perpetually be preserved, have decided to ornament it with such noble pictures that if it comes in the hands of one who is lacking such understanding, he will preserve it for the beauty of the pictures.« (Übersetzung nach O. Gombosi, ebda., S. XC).

41 H. Neusidler, Lautenbuch 1536a (wie Anm. 26), fol. a ir.

42 H. Neusidler, Lautenbuch 1536b (wie Anm. 28), fol. A i ${ }^{\mathrm{r}}$. 
zu einzelnen Stücken keinen Zweifel an seiner Motivation und dem Zweck der Intavolierungen: Sein Druck ist klar auf einen Käuferkreis zugeschnitten, und Neusidler versäumt auch keine Gelegenheit, sein Produkt entsprechend anzupreisen. Das erste Buch ist fast wie ein Lauten-Kurs aufgebaut und beginnt mit Erläuterungen für den Anfänger zu Besaitung, Stimmung und Haltung der Laute sowie Erklärungen zu Tabulatur und Fingersätzen. Sodann folgen einfache Übungen zur Ausbildung der Finger und des Tabulaturlesens, bis schließlich an die Intavolierungen gegangen wird, die mit sehr schlichten, zweistimmigen Bearbeitungen anfangen und schließlich zu den dreistimmigen fortschreiten - jeweils zunächst mit ausnotiertem Fingersatz, später nur noch mit Hilfen für besondere Stellen. Der zweite Band enthält eine Materialsammlung für den fortgeschrittenen Lautenisten mit dreistimmigen Intavolierungen, die Neusidler zusätzlich »nach Lutanistischer vnd auch Organistischer art [gesetzt] ${ }^{43}$ das heißt $»$ mit leufflein vnd Coloraturen gezyret «, ${ }^{44}$ kurzum instrumentenspezifisch arrangiert hat.

In seinen beiden Lautenbüchern finden sich dabei etliche Stücke in mehrfachen Bearbeitungen, zum Beispiel im Schülerbuch einmal in zweistimmiger, später in dreistimmiger Fassung und gelegentlich im zweiten Buch dann nochmals in dreistimmiger, aber stark verzierter Version. Diese Verfahrensweise, obwohl bei Neusidler aus eindeutig didaktischen Motiven eingesetzt, scheint einer Aufführungspraxis entnommen zu sein, die in Hans Gerles Tabulatur auf die Laudten von 1533 in der Beschreibung von Adolf Blindhamers Spiel bestätigt wird:

... so er zu einem gesatzten stücklein gegriffen / hat er das erstlich / wie es in noten gestanden / mit wenig Coloraturen / zum andern mit wolgestelten leuflein geziert / vnd zum dritten durch die Proportion geschlagen vnd volfürt / Vnd doch einer solchen gestalt / damit der süssigkait vnd vollkomenheyt des gesangs nichts benomen worden ist. ${ }^{45}$

Ein solches Stück, das in beiden Büchern und in unterschiedlichen Schwierigkeitsgraden vorliegt, wird in der zweiten, komplexeren Bearbeitung als $»$ Alex. agricola. Ander nacken vp dem Rhin $\aleph^{46}$ bezeichnet. Leider handelt es

43 H. Neusidler, Lautenbuch 1536a (wie Anm. 26), fol. a iiir ${ }^{r}$ - bemerkenswert ist die offenbare Orientierung der Verzierungskunst an der Praxis der Organisten.

44 Ebda.

45 Hans Gerle, Tabulatur auff die Laudten, Nürnberg 1533, fol. $\mathrm{II}^{\mathrm{v}}$, zitiert nach: Frühe Lautentabulaturen im Faksimile, hrsg. von Crawford Young und Martin Kirnbauer, Winterthur 2003 (Pratica Musicale, 6), S. 249f.

46 H. Neusidler, Lautenbuch 1536b (wie Anm. 28), fol. Q iiv $-\mathrm{P} \mathrm{i}^{\mathrm{v}}$. Neusidler bemerkt im Anschluss an den Abdruck: »Welchem der Tannernack zu schwer ist / der findet jhn in 
sich dabei aber nicht um Agricolas bekanntes Tandernaken und muss von daher aus der Liste der Lautenbearbeitungen seiner Stücke gestrichen werden. Eine weitere Intavolierung, die Neusidler Alexander Agricola zugeschrieben hat, das Ave sanctissima, ${ }^{47}$ lässt sich bislang auch durch keine Parallelüberlieferung bestätigen, so dass ihr vorerst das gleiche Schicksal vorbehalten bleibt.

Ein Stück jedoch, das im Druck als Bearbeitung von Jacob Obrechts »Si dedero« ausgegeben wurde, ist in Wahrheit das »Si dedero « Agricolas. ${ }^{48}$

Damit liegt in jeder der drei Tabulatursammlungen jeweils eine Fassung dieser Komposition vor und bestätigt die Beliebtheit dieses Stückes, das von allen Kompositionen Agricolas die häufigste Überlieferung erfahren hat. ${ }^{49}$ Durch diese dreifache Bearbeitung bietet sich ferner die Möglichkeit eines direkten Vergleichs der Fassungen untereinander, um festzustellen, wie die Autoren dieser sehr unterschiedlichen Quellen jeder auf seine Art mit der gleichen Vorlage umgangen sind.

Es fällt zunächst auf, dass alle drei Bearbeiter ihrer Vorlage sehr eng gefolgt sind. Der synoptische Abdruck des Originals mit den drei Lautenquellen zeigt, dass sie sich bis zur Schlusskadenz taktgenau decken. Lediglich in der Version Neusidlers gibt es einen kleinen Überhang, in dem der Lautenist $>$ überschüssige Energie< in Form von Skalenläufen auf dem Schlussklang gewissermaßen >abarbeiten< kann (siehe Notenbeispiel 4a).

Notenbeispiel 4a-f (auf den folgenden Seiten): A. Agricola, "Si dedero «, Synopse der Vorlage mit den Versionen von Spinacino, Capirola und Neusidler a) T. 75-Ende, b) T. 1-8, c) T. 10f., d) T. 66-70, e) T. 15, f) T. 23f., g) T. 51f.

meinem getruckten schüler bůch ein wenig ringer / vnd sehr gut.« Die schlichte Bearbeitung siehe H. Neusidler, Lautenbuch 1536a (wie Anm. 26), fol. q iir ${ }^{\mathrm{r}} \mathrm{q}$ iiiir ${ }^{\mathrm{r}}$.

47 H. Neusidler, Lautenbuch 1536b (wie Anm. 28), fol. I ii ${ }^{\mathrm{v}}-\mathrm{K}$ iii $^{\mathrm{r}}$.

48 Vgl. Harmonice Musices Odhecaton A, hrsg. von Helen Hewitt, Cambridge Mass. 1942 (Publications of the Mediaeval Academy of America, 42), Nr. 56, sowie O. Gombosi, Compositione, wie Anm. 32, S. LXXXV, und Howard Mayer Brown, Instrumental Music Printed Before 1600. A Bibliography, Cambridge, Mass. $\left({ }^{1} 1965\right),{ }^{2} 1967$, S. 55.

49 Edward Lerner listet in seiner Edition 21 Überlieferungsträger - ohne Einbeziehung der Instrumentalbearbeitungen - und kommentiert: »By far Agricola's most famous work « (wie Anm. 21, S. XVI). Eine Wertschätzung von Agricolas Komposition findet sich durch die dem Titel beigestellte Anmerkung im Index des Capirola-Tabulaturbuches: »Si dedero. Canto belisimo mai esta sona asta foza«. Die Anmerkung könnte in etwa gedeutet werden als $\gg$ Si dedero: Canto bellissimo - mai questo suono ha avuto questa foggia“, übersetzt ungefähr $\gg$ Si dedero: Ein sehr schönes Lied - nie hat diese Melodie eine vergleichbare Form gehabt«. (Ich danke Lorenza Donadini für diesen Erklärungsansatz.) 


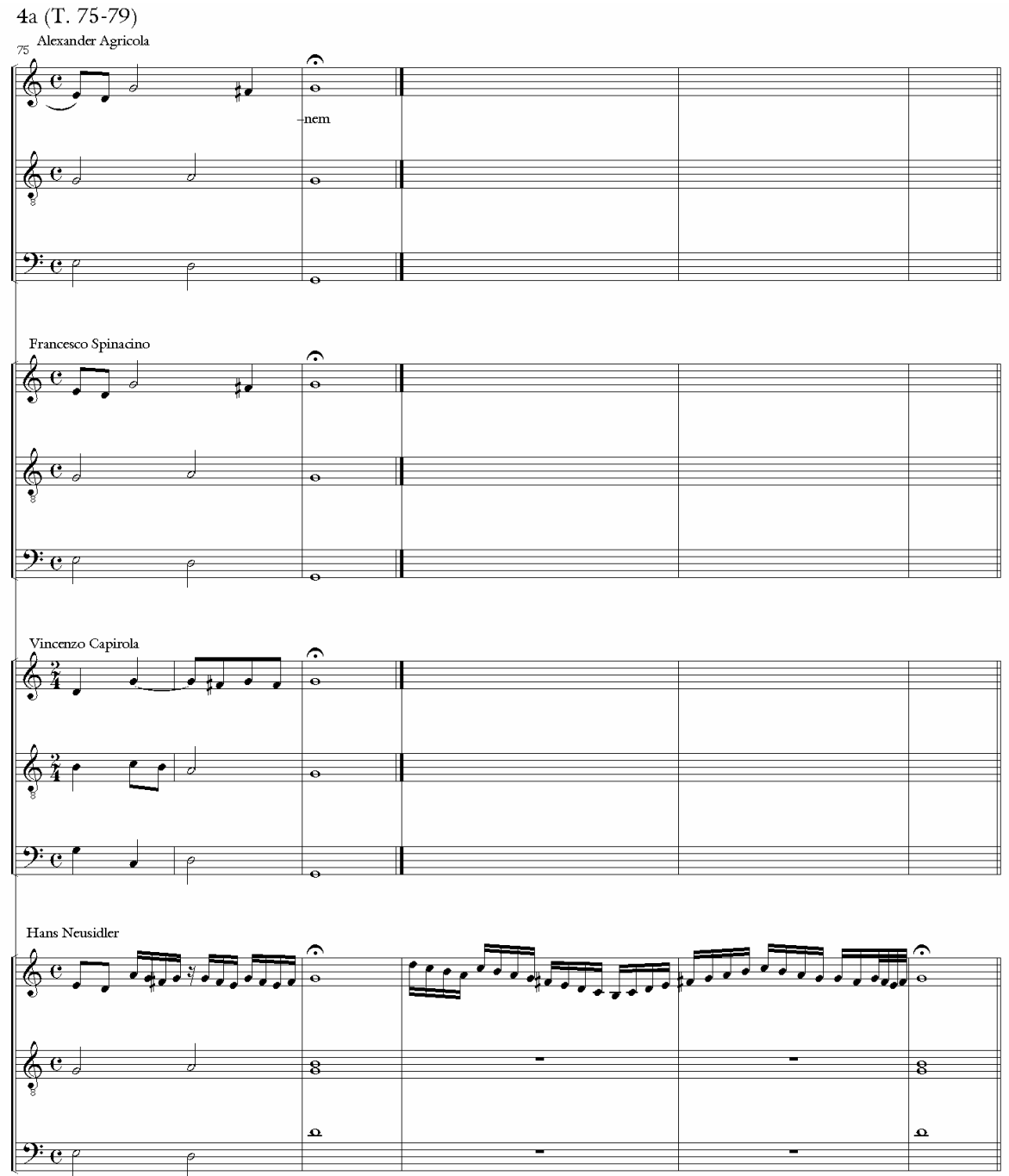

In einigen Punkten unterscheiden sich die drei Tabulaturen schon rein äußerlich: bei Spinacino und Neusidler sind die Mensurstriche pro ganze Note (einer Brevis im Original) in der Tabulatur gesetzt, während bei Capirola eine Ebene feiner unterteilt wird - diese Tendenz zeigt sich in den CapirolaTabulaturen generell. Die Taktnummerierung in der synoptischen Übertragung orientiert sich dabei an der gröberen Unterteilung der anderen beiden Überlieferungen. Ein weiterer Aspekt, der beim Ausprobieren auf dem 
$4 \mathrm{~b}$ (T. 1-8)
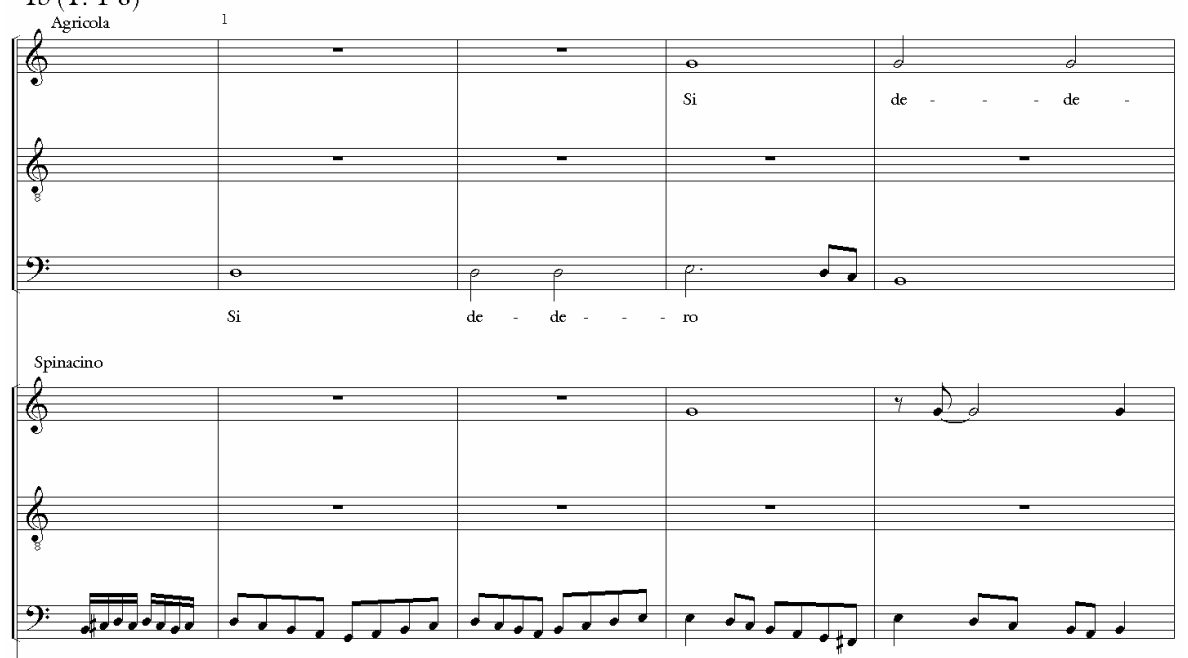

Capirola
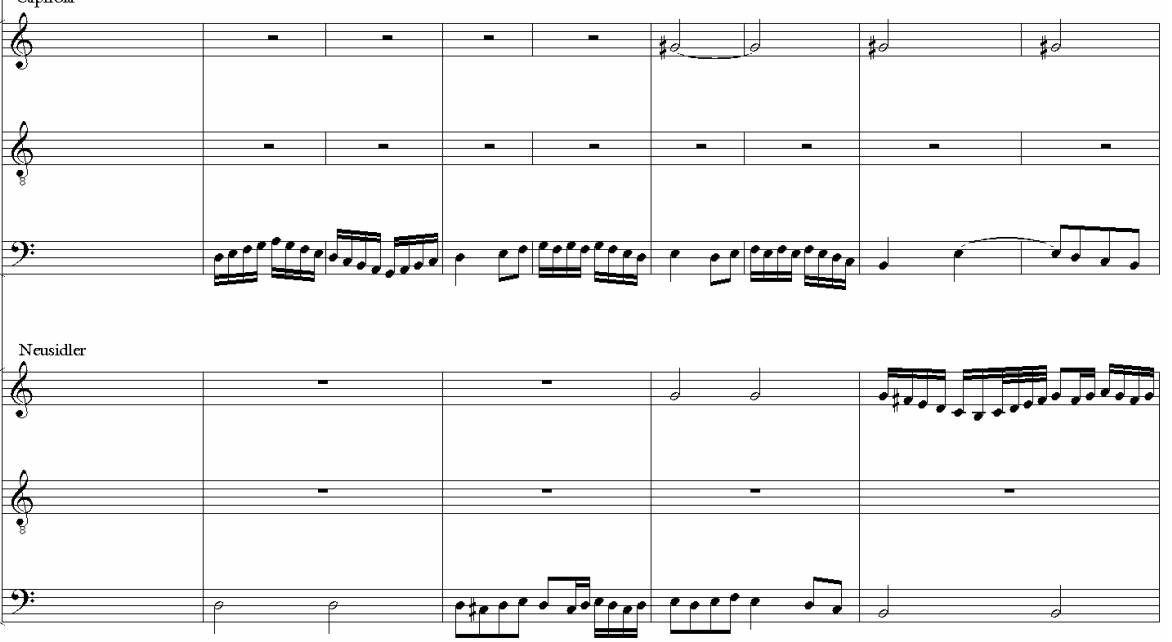

Instrument sofort auffallt, ist die relative Transposition der Komposition: In jeder der drei Tabulaturen wurde eine andere Lage gewählt. Spinacinos Intavolierung liegt erwartungsgemäß am höchsten und fordert den Interpreten vor allem dadurch; Capirola setzt das Stück einen Ton und Neusidler gar eine Quarte tiefer als Spinacino. Für den Abdruck hier wurden alle Übertragungen jedoch auf die Tonhöhe der Vorlage transponiert, um die Vergleichbarkeit der Versionen zu erleichtern. 


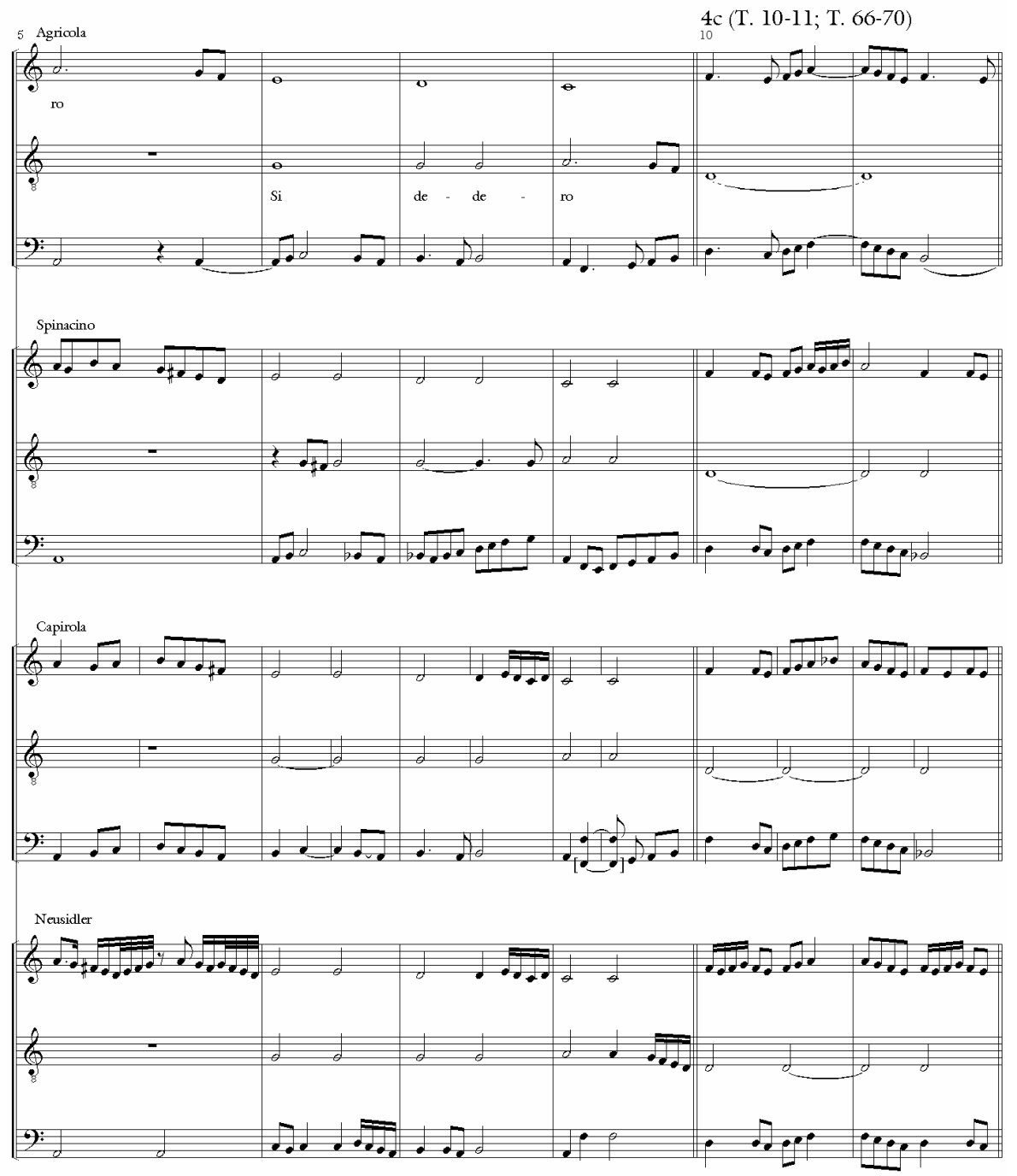

In der Wahl der Diminutionsebene wirkt die zeitliche Abfolge der Tabulaturen fast wie eine Steigerung: Spinacino diminuiert relativ gesehen am langsamsten, indem er - von seltenen Ausnahmen abgesehen - die AchtelnotenEbene wählt. Capirola und Neusidler diminuieren häufiger auf der SechzehntelEbene und Neusidler neigt am ehesten dazu, bestimmte Floskeln noch eine Ebene tiefer bei den Zweiunddreißigsteln anzusiedeln. Diese extrem schnellen Verzierungen beschränkt er aber ganz klar auf Viertongruppen im Skalenlauf, 


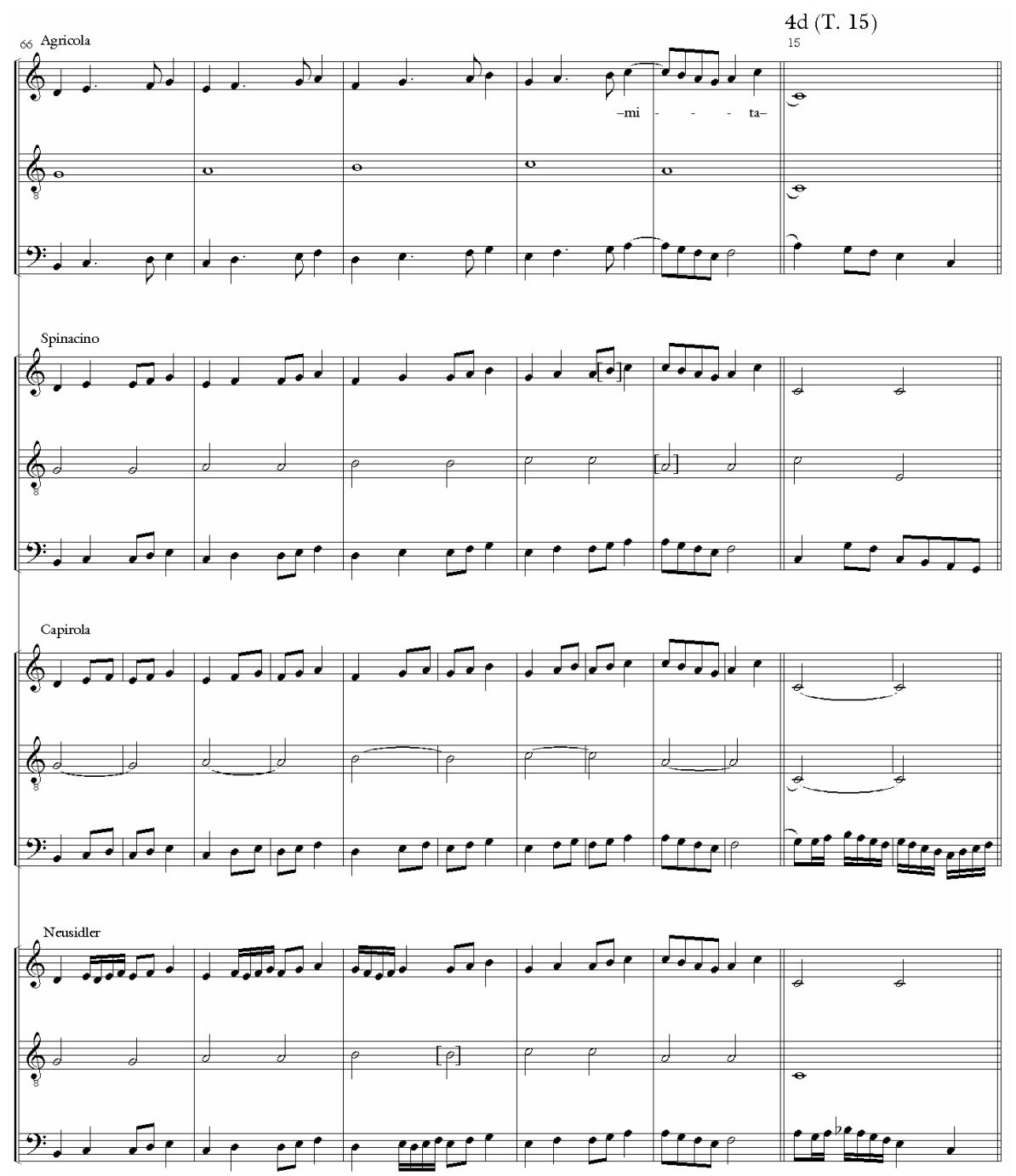

so dass diese Figuren sehr geregelt und idiomatisch auftreten.

Ein neuralgischer Punkt bei der Intavolierung ist die Behandlung langer Notenwerte einer gegebenen Vorlage, insbesondere wenn sie dort von struktureller Bedeutung sind, wie beispielsweise in der Imitation des langsamen Motivs, mit der das »Si dedero« eröffnet wird (siehe Notenbeispiel $4 \mathrm{~b}$ ).

Gegenüber einer vokal besetzten Fassung würden die Intavolierungen des Stückes in der Aufführungsgeschwindigkeit aufgrund der vielen Diminutionen 


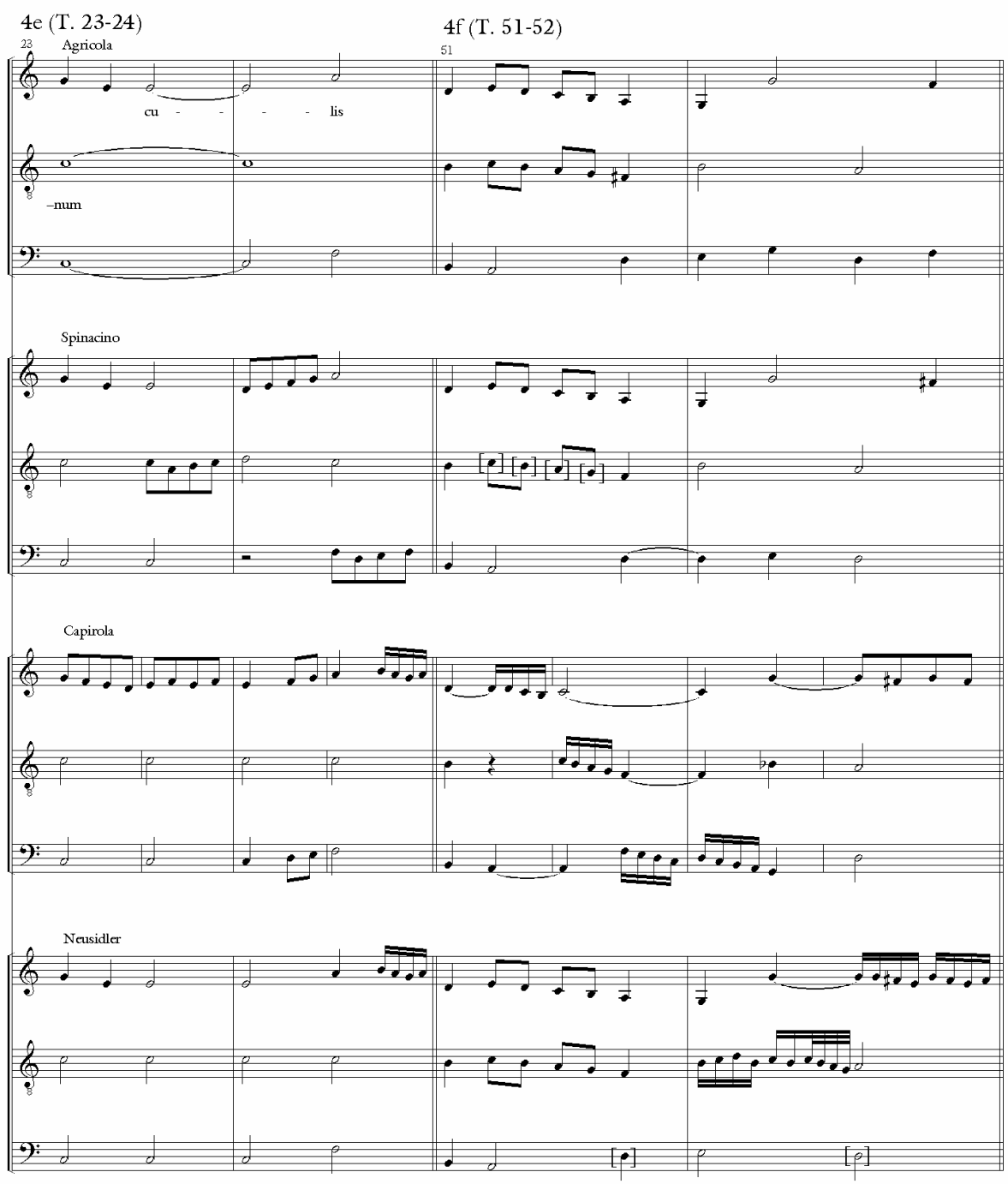

sicher langsamer angelegt werden. Gerade die lang ausgehaltenen Töne, die ohnehin mit einer Laute schlecht dargestellt werden können, werden dann noch nachteiliger für das Instrument. An diesen Stellen begegnet man in allen Tabulaturen regelmäßig und wenig überraschend der oben schon erwähnten Aufspaltung in kleinere Notenwerte. Hinzu tritt die auch angewandte Möglichkeit der Umspielung und Auszierung langer Noten der Vorlage. Beide Techniken werden gerade zu Anfang der Komposition von allen 
drei Tabulaturen genutzt. Während die Versionen von Spinacino und Capirola sich jedoch in den ersten beiden Takten in quasi-präludierenden Läufen ergehen, beginnt Neusidlers Fassung mit einem Motiv, das in der Folge imitiert wird und damit die Struktur der Vorlage stärker widerspiegelt. Capirola hebt immerhin den zweiten Einsatz des Motivs deutlicher hervor und ermöglicht somit die Imitation zwischen Cantus und dem zuletzt einsetzenden Tenor (Takt 3-8), wobei die Wahl der Musica ficta für den Cantuseinsatz (in der Transkription ein gis) vor allem in Bezug auf das unmittelbar darauf mit $g$ einsetzende Motiv im Tenor bemerkenswert ist. ${ }^{50}$ In Spinacinos Fassung ist der imitierende Beginn hingegen fast völlig verschleiert - dieses formgebende Element der Vorlage scheint für seine Bearbeitung keine große Rolle gespielt zu haben. Mit dem nächsten SoggettoEinsatz in Takt 16 verlagert sich dieser Eindruck auch auf die anderen Intavolierungen. Selbst Neusidler hebt nur noch die zweite und dritte Imitation hervor, während Capirola diese Struktur wie Spinacino gänzlich ignoriert. Anhand dieser Beobachtungen drängt sich die Vermutung auf, dass die Intavolierungen, vielleicht aufgrund einer Verlangsamung des Gesamttempos der Komposition, sich eher auf kleinere Strukturen konzentrieren und größere formale Kriterien an Bedeutung verlieren.

Dieser Eindruck bestätigt sich, wenn man den Blick auf Passagen wendet, die schon in der originalen Fassung Agricolas >ohrenfällig< sind, besonders die Dezimparallelen und Sequenzen, beispielsweise in Takt 10f., 25-29, 32f., 60f. und 66-70 (siehe Notenbeispiel 4c). An solchen Stellen ähneln sich die verschiedenen Fassungen sehr stark. Auch auffällige, bewegte Motive der Vorlage finden in der Regel eine direkte Abbildung in den Lautenfassungen, zum Beispiel Takt 40-44 oder die Brücken nach Kadenzen im Bassus der Takte 15, 53f. und 64 (siehe Notenbeispiel 4d).

Eine Sensibilität für bedeutsame Punkte in der Komposition kann man am Beispiel von Takt 23f. beobachten (siehe Notenbeispiel 4e). Die Vorlage Agricolas kommt hier zur Ruhe, stagniert fast ein wenig, bevor sie sich zur groß angelegten Kadenz zum Ende des ersten Teils hin aufschwingt. Alle

50 Gombosi kommentiert zum Einsatz von Akzidentien bei Capirola: »Capirola's transcriptions offer highly interesting cases of controversial accidentals. Even in imitations, false cross-relations are not lacking. Such things do occur in more carelessly transcribed versions, where the notes of the original are quite mechanically translated into the figures of lute tablature. Yet the cases we have mentioned above, while daringly nonconforming to theoretical standards as we understand them, are hardly the results of careless intabulation.«(O. Gombosi, Compositione, wie Anm. 32, S. XXIX). 
Intavolierungen, besonders aber die Neusidlers, folgen dieser Vorgabe und gehen die Diminution in diesen Takten wesentlich reduzierter an.

In der Intavolierung Hans Neusidlers lässt sich eine Tendenz feststellen, die Vorlage polyphoner abzubilden, als in den anderen Tabulaturen. Nicht allein die oben erwähnte stärkere Betonung der imitativen Passagen der Vorlage, sondern auch der Einsatz von Verzierungen bestätigt dieses Ansatz, wie im Takt 52 zwischen Tenor und Cantus (siehe Notenbeispiel 4f).

Die Intavolierung von Capirola hingegen neigt eher $\mathrm{zu} »$ bastarda«Diminutionen, die sich durch die Stimmen hinwegbewegen, anstatt jede Stimme separate Verzierungsmotive ausführen zu lassen. Sehr gut kann man diese Neigung in Takt 51 beobachten, wo Spinacino lediglich die Cantusmelodie übernimmt, Neusidler den Kontrapunkt praktisch identisch abbildet und Capirola überlappende Läufe durch alle Stimmen zieht (siehe Notenbeispiel 4f).

Der Vergleich der drei Bearbeitungen von Agricolas $\gg$ Si dedero« zeigt: Was idiomatische Wendungen angeht, so ähneln sich die drei Tabulaturen in vielen Fällen - besonders bei bewegten Stellen des Originals folgen die Intavolierungen der Vorlage recht eng und verwenden typische Floskeln, um den Satz für die Laute sinnvoll abzubilden. Die Wahrnehmung von Strukturen der Vorlage und die Einschätzung ihrer Bedeutung hingegen ist von Lautenist zu Lautenist sehr unterschiedlich und tritt in den individuellen Bearbeitungen klar zu Tage. Im Grunde sind es vor allem Passagen mit lang ausgehaltenen Tönen, die von den Bearbeitern bisweilen sehr unterschiedlich in die Tabulatur übersetzt und variiert werden.

Die genannten Beobachtungen reichen aus, dass sich ein heutiger Lautenist die vorhandenen Tabulaturen für seine Zwecke einrichten oder seine eigenen Solofassungen auch von Agricola-Kompositionen schaffen kann Fassungen, die stilgerecht sind, zugleich aber individuell auf die Fertigkeiten des Intavolierenden abgestimmt. Dabei sind der Repertoireauswahl praktisch keine Grenzen gesetzt - wie wir schon an den frühesten Intavolierungen sehen können, finden Chansons, Motetten und textlose Kompositionen, selbst Messteile gleichermaßen Eingang in die Intavolierungspraxis.

\section{Die Laute im professionellen Lautenduo}

Allein aus der Tatsache, dass weder Spinacino noch Capirola ihren Intavolierungen Komponistenzuweisungen beigeben und bei Neusidler einige Autoren den Stücken falsch zugeordnet sind, ist ersichtlich, dass Agricola im Prinzip keine gesonderte Behandlung bei der Umsetzung auf der Laute er- 
fährt: seine Kompositionen stehen in einer Reihe mit den Werken seiner Zeitgenossen. Nur bei einem Stück scheint Neusidler eine Ausnahme zu machen. Seiner Intavolierung des Cecus non judicat de coloribus im zweiten Lautenbuch (für den erfahrenen Spieler) stellt er folgende Beischrift voran:

Hie folget ein stuck / das hat zwen theil / vnd wirt von den alten singern der Alexander genant / vnd ist sehr fast kunstreich / vnd sein fast schier alle clausen der Musica darin begriffen / ist auch seer gerad vnd scharpff colorirt / mit der aller besten Organistischen coloratur oder laiflein / als einer sein leben lang der art noch nie gesehen hat / das wirt sich in dem werck erfinden. ${ }^{51}$

Es ist bemerkenswert, wie hoch Neusidler noch dreißig Jahre nach Agricolas Tod dessen Kompositionen einschätzt und sie seinem Publikum empfiehlt. Die besondere Wertschätzung, die dieses Stück bei Neusidler erfährt, ist erstaunlich. Es wird mit dem Namen des Komponisten selbst identifiziert, Agricola >ist< sein Cecus. Und auch dieses Stück findet sich als zweistimmige Version und in schlichterer Fassung in Neusidlers Schülerbuch. ${ }^{52}$ Verblüffend ist nun die Feststellung, dass Neusidler den originalen dreistimmigen Satz nicht auf einen kompositorischen Kern von Cantus und Tenor reduziert, sondern als >Schülerfassung <enor und Contratenor zusammenzieht. Diese Vereinfachung auf einen zweistimmigen Satz bezeichnet er wiederholt selbst als $»$ nur ein[en] ausklaubten kern $\ll^{53}$ und wendet sie offensichtlich aus rein didaktischen Gründen an. Zusätzlich transponiert er seine Reduktion des Cecus um eine Quarte nach oben. Ein Vergleich von anderen zweistimmigen Bearbeitungen für den Schüler mit ihren jeweiligen Vorlagen bestätigt die Diagnose des Cecus-Satzes: Neusidler reduziert für die zweistimmige Bearbeitung in jedem einzelnen Fall den Satz auf die Unterstimmen aus Tenor und Contratenor bassus - auch bei ursprünglich vierstimmigen Sätzen. Zusätzlich transponiert er den Satz jedes Mal um eine Quarte nach oben. Die Transposition wird offenbar vorgenommen, um den »ausklaubten kern« in eine angenehme Mittellage auf dem Instrument zu bringen, während für die späteren dreistimmigen Versionen der gleichen Lieder (weiter hinten im Schülerbuch und im zweiten Teil für den erfahrenen Lautenisten) mit hinzugefügtem Cantus die ursprüngliche Lage wiederhergestellt wird - zweifellos um den Cantus noch bequem unterbringen zu können.

Die Transposition und die Reduktion sind ganz klar Eingriffe, die Neusidler vornimmt, um die Sätze für den Anfänger auf der Laute so ein-

51 H. Neusidler, Lautenbuch 1536b (wie Anm. 28), fol. P iv .

52 H. Neusidler, Lautenbuch 1536a (wie Anm. 26), fol. g iiiiv - h iiiiv ${ }^{\mathrm{v}}$.

53 H. Neusidler, Lautenbuch 1536a (wie Anm. 26), fol. fiir ${ }^{r}$. 
fach und angenehm wie möglich zu gestalten. Warum er aber nicht Cantus und Tenor intavoliert, sondern Tenor und Contratenor bassus, ist besonders bei den Liedintavolierungen, die noch stark auf diesem Gerüst beruhen, weniger leicht nachvollziehbar. Vielleicht war die enge Lage beider Stimmen und die damit verbundene leichtere Umsetzung auf der Laute ausschlaggebend für Neusidlers Wahl. Dagegen spricht jedoch, dass sich in diesen Sätzen auch der Cantus in der Regel nicht sehr weit vom Tenor entfernt. Der Beweggrund mag von daher didaktischer Natur gewesen sein, zumal die bewusste Auswahl von sehr bekannten Liedern das Erlernen auf der Laute nach Neusidlers eigener Aussage erleichtern sollte $e^{54}$ - die Wahl der intavolierten Stimmen hingegen könnte sich aus anderer Motivation heraus ergeben haben: Tradition.

Wenn man das professionelle Lautenduo als eine Standardbesetzung für instrumentale Ausführung von komponierten Vorlagen auf der Laute im 15. Jahrhundert annimmt, dann könnte es sein, dass, wenn man in dieser Zeit nur einen Teil einer Komposition auf der Laute intavolieren wollte, man automatisch in die reinfacherere Rolle innerhalb dieses Duos verfiel, nämlich die des Tenorista. Auch die Transposition in eine Mittellage des Tenor-Instrumentes ergäbe dann Sinn, denn der Improvisateur für die Oberstimme spielte ja gewöhnlich auf einem kleineren Instrument, auf dem der Cantus auch in höherer Lage noch problemlos ausführbar gewesen sein dürfte.

Und tatsächlich scheint es sich bei Neusidlers Behandlung der Unterstimmen von Cecus non judicat de coloribus um das gleiche Prinzip zu handeln, das zuvor Spinacino schon in seinen Duetten verwandte: Tenor und Contratenor sind auf sehr ähnliche Weise eingerichtet, während die fehlende Oberstimme sich gut in Form von improvisierenden Diminutionen ergänzen lässt. Es ist klar, dass dies nicht die Absicht Neusidlers war und auch ein hinzutretender professioneller Lehrer, der diese Oberstimme für den Tenorista-Schüler hätte zur Verfügung stellen können, kann nicht angenommen werden, denn Neusidler betont in seinem Vorwort ausdrücklich, dass kein Lehrer für sein Lautenbuch benötigt würde (wobei solche >Werbesprüche< auch reinem Verkaufskalkül geschuldet sein könnten). Es scheint aber so, dass in der Art der Intavolierung noch eine Praxis nachscheint, die für die Umsetzung solcher Musik auf der Laute weit verbreitet gewesen sein muss.

54 Neusidler schreibt dazu: „Hie folgen noch mer ettlicher guter alter lieder ... Die hab ich darumb gesetzt / das sie vorhin in vil landen weyt vnd preit erkannt / vnd vil dester leichter zu lernen sind weder die vnerkanten" $(\mathrm{H}$. Neusidler, Lautenbuch 1536a, wie Anm. 26, fol. $\mathrm{fi}^{\mathrm{v}}$ ). 


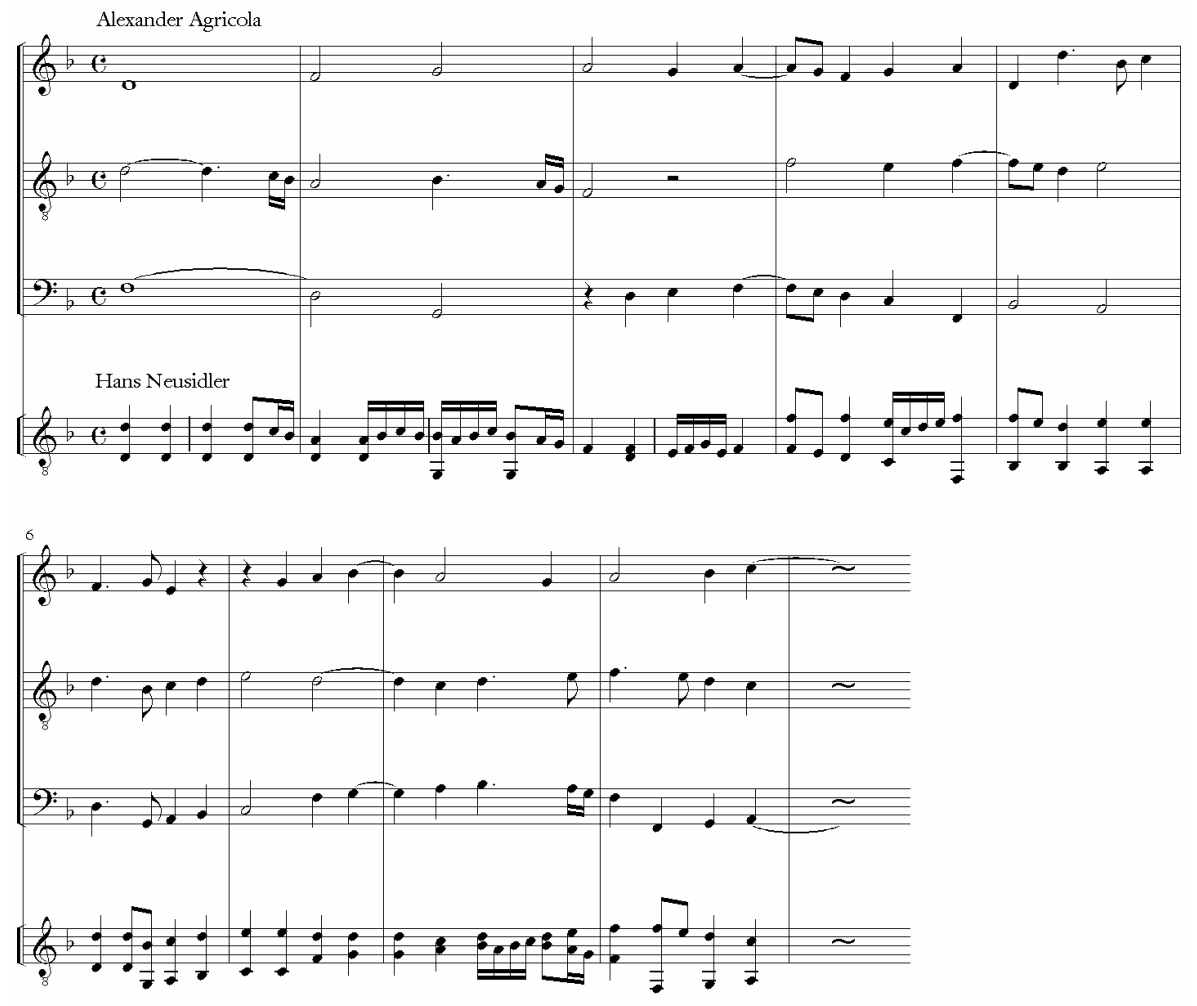

Notenbeispiel 5: A. Agricola, Cecus non judicat de coloribus (Anfang), oben: Vorlage, unten: Intavolierung von $\mathrm{H}$. Neusidler, Alexander agricola

Und dadurch ergibt sich mit dem Duo eine weitere Möglichkeit für den Einsatz von Lauten in Kompositionen um 1500 - mit einer klaren Rollenverteilung: Der Tenorista ist zuständig für eine Einrichtung der Unterstimmen der Vorlage, während der Solist die Oberstimme in einem bestimmten Stil improvisierend diminuiert. Für einen ersten Versuch in diese Richtung bietet sich von daher Neusidlers vorbereitete Intavolierung des >Unterbaus< von Cecus non judicat de coloribus an. Zu Neusidlers Zeit wäre hier wahrscheinlich stilistisch eine >modernere< Art der Oberstimmenverzierung angewandt worden als es um 1500 noch der Fall gewesen wäre; mit den Spinacino-Diminutionen existiert aber zumindest ein überliefertes Beispiel für eine Praxis, die mit Sicherheit ins 15. Jahrhundert zurückweist. Als Abschluss des Referats beim Symposium am 28. April 2006 in der Musikhochschule Trossingen wurde nach Art von Notenbeispiel 6 eine Version 
des Cecus non judicat de coloribus mit zwei Lauten präsentiert, die diese Beobachtungen vereint. Als Modell für die Oberstimmenauszierung wurden die Duos von Spinacino verwandt, besonders das Jay pris amours, um eine mögliche Oberstimmenversion zu Neusidlers Cecus-Intavolierung der Unterstimmen zu verfassen. ${ }^{55}$
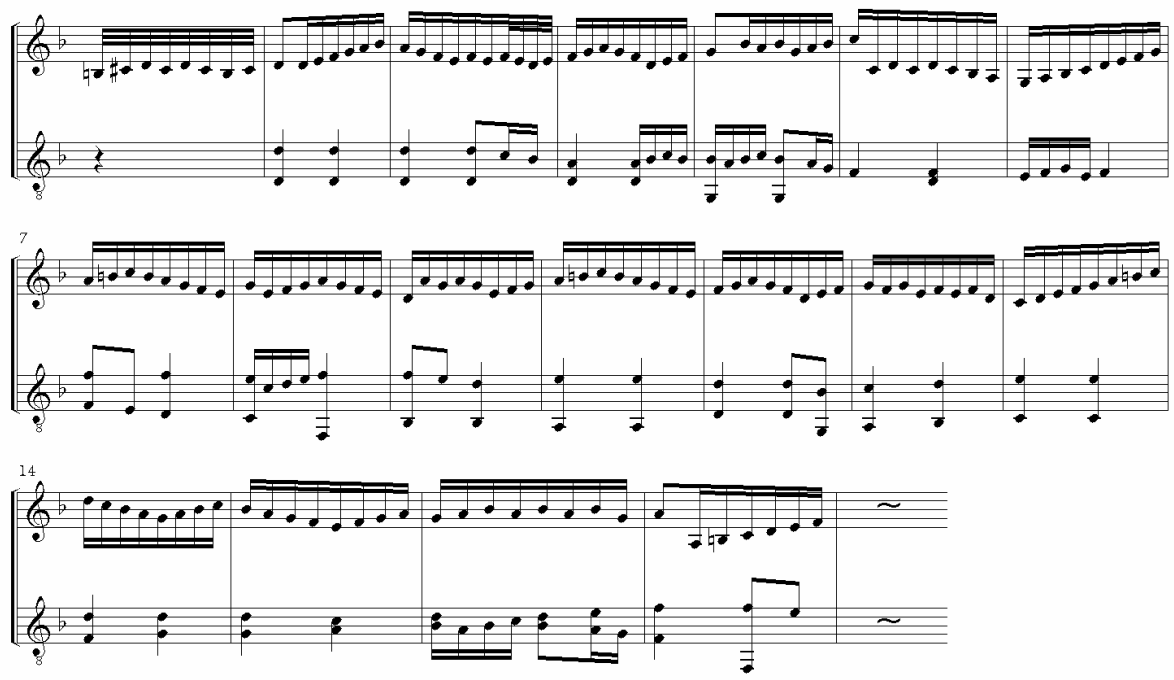

Notenbeispiel 6: A. Agricola, Cecus non judicat de coloribus (Anfang), hypothetische Fassung für Lautenduo (Begleitung: Hans Neusidler, Diminution: Marc Lewon). Im Verlauf der Fassung wird der gesamte Tonumfang der Diminutionslaute vom tiefsten bis zum höchsten möglichen Ton ausgenutzt.

55 An dieser Stelle möchte ich mich ganz besonders bei meinem Lehrer Crawford Young bedanken, auf dessen langjähriger Arbeit und zahlreichen Anregungen dieser Vortrag fußt und der so freundlich war, sich als Tenorista zur Verfügung zu stellen. 